\title{
Thermo-dynamic and chemical properties of the intra-cluster medium
}

\author{
A. Leccardi ${ }^{1,2}$, M. Rossetti ${ }^{2}$, and S. Molendi ${ }^{2}$ \\ 1 Università degli Studi di Milano, Dip. di Fisica, via Celoria 16, 20133 Milano, Italy \\ e-mail: silvano@iasf-milano.inaf.it \\ 2 INAF-IASF Milano, via Bassini 15, 20133 Milano, Italy \\ Received 8 August 2009 / Accepted 22 October 2009
}

\section{ABSTRACT}

\begin{abstract}
Aims. We aim to provide constraints on evolutionary scenarios in clusters. One of our main goals is to understand whether, as claimed by some, the cool core/non-cool core division is established once and for all during the early history of a cluster.

Methods. We employ a sample of $\simeq 60$ objects to classify clusters according to different properties. We characterize cluster cores in terms of their thermo-dynamic and chemical properties and clusters as a whole in terms of their dynamical properties.

Results. We find that I) the vast majority of merging systems feature high-entropy cores (HEC) and II) objects with lower entropy cores feature more pronounced metallicity peaks than objects with higher entropy cores. We identify a small number of medium (MEC) and high (HEC) entropy core systems that, unlike most other such objects, feature a high central metallicity. The majority of these outliers are mergers, i.e. systems far from their equilibrium configuration.

Conclusions. We surmise that medium (MEC) and high (HEC) entropy core systems with a high central metallicity have recently evolved from low-entropy core (LEC) clusters that have experienced a heating event associated to AGN or merger activity.
\end{abstract}

Key words. X-rays: galaxies: clusters - galaxies: clusters: general - galaxies: abundances

\section{Introduction}

The classification of objects is an important step in constructing viable physical models. This is particularly true for disciplines like astrophysics where the impossibility of performing measurements under a set of rigorously controlled conditions entails that evolutionary schemes are inferred primarily by comparing properties observed in different objects. Galaxy clusters are no exception to this rule, as for other astrophysical sources, much of the early work has concentrated on establishing a taxonomical framework. In the optical band, classification schemes are based on the richness (Abell 1958; Zwicky et al. 1968) and on the morphological properties that have been found to correlate with the dynamical state of the systems (Abell 1965, 1975). In X-rays, classification attempts are generally focused on core properties for the rather obvious reason that cores are the regions more easily accessible to observations. Most workers concentrate on defining indicators that distinguish between cool core (hereafter $\mathrm{CC}$ ) and non-cool core (hereafter NCC) systems; these indicators are typically based on estimates of the intensity of the surface brightness peak (Vikhlinin et al. 2007), the temperature (e.g. Sanderson et al. 2006), the cooling time (e.g. Baldi et al. 2007), or the entropy (e.g. Cavagnolo et al. 2009) of the central regions of clusters. There have been attempts to derive dynamical properties of cluster ensembles from X-rays, the best known example being perhaps that of the power-ratio technique (Buote \& Tsai 1995, 1996). Comparison of core with morphological properties, as identified with the power-ratio technique, shows that more disturbed systems tend to have less defined cores (Buote \& Tsai 1996; Bauer et al. 2005). One should, however, keep in mind that the characterization of the degree of relaxation of clusters through X-ray morphology is limited by projection effects (e.g. Jeltema et al. 2008) and that the limited amount of information available at large cluster radii, beyond $\simeq 0.2 R_{180}$, provides a further complication. There have been some attempts to compare dynamical and core properties on individual objects (e.g. A2034, Kempner et al. 2003), sometimes using information from different energy bands (e.g. A1644, Reiprich et al. 2004).

Classification schemes based on chemical properties of clusters have enjoyed considerably less attention. We have known for some time now that CC clusters, formerly known as coolingflow clusters, feature significant central abundance excesses (De Grandi \& Molendi 2001) and that the amount of iron associated with these excesses is consistent with being produced from the BCG galaxy invariably found at the center of CC systems (De Grandi et al. 2004). Interestingly, there has been no attempt so far to classify clusters by simultaneously making use of thermo-dynamical and chemical properties. In this paper we employ a medium-size sample, $\simeq 60$ objects, to address the issue of cluster classification from various angles, more specifically we 1) define an entropy indicator to classify cluster cores with respect to outer regions; 2) provide a dynamical classification based on radio, optical, and X-ray properties; 3) compare core and dynamical properties; 4) compare, for the first time, the entropy based classification with a chemical classification. As we shall see, our classification work will allow us to gain considerable insight into how cluster dynamical, thermo-dynamical, and chemical properties relate to each other. Another interesting result that will emerge from our analysis is the relevance of outliers, i.e. objects that fall outside of the distributions defined by the majority of systems, in constraining the evolutionary processes that shape galaxy clusters.

The breakdown of the paper is the following. In Sects. 2 and 3 we respectively describe the sample selection and the data analysis. In Sect. 4 we provide an account of how our entropy and cooling time indicators have been constructed. In Sect. 5 
we describe two classification schemes based on core and dynamical properties, while in Sect. 6 we compare them with the entropy-based classification scheme defined in Sect. 4. In Sect. 7 we define a classification scheme based on chemical properties and compare it to the other classification schemes discussed in the paper. Finally in Sect. 8 we summarize our main findings.

Quoted confidence intervals are $68 \%$ for one interesting parameter (i.e. $\Delta \chi^{2}=1$ ), unless otherwise stated. All results assume a $\Lambda$ CDM cosmology with $\Omega_{\mathrm{m}}=0.3, \Omega_{\Lambda}=0.7$, and $H_{0}=70 \mathrm{~km} \mathrm{~s}^{-1} \mathrm{Mpc}^{-1}$.

\section{The sample}

Starting from the XMM-Newton archive, we selected a sample of hot clusters $(k T>3 \mathrm{keV})$. The redshift spans between 0.03 and 0.25 , and the galactic latitude is greater than $20^{\circ}$. Among those clusters satisfying the above selection criteria, we retrieved all observations performed before March 2005 (when the CCD6 of EPIC MOS1 was switched off ${ }^{1}$ ) and available by the end of May 2007. In Table 1 we list the observations of all the clusters we analyzed and report cluster physical properties (e.g. redshift and temperature) and observational details (e.g. total exposure time and filter). The redshift value (from optical measurements) was taken from the NASA Extragalactic Database ${ }^{2}$ and $k T_{\text {OUT }}$ was derived from our analysis (see Sect. 3.2). Observations were performed using THIN1 or MEDIUM filters. We excluded from the sample observations that are badly affected by soft proton flares, so that the total (i.e. MOS1+MOS2+pn) exposure time for all observations is at least $20 \mathrm{ks}$. We also excluded observations of extremely disturbed clusters for which it was impossible to define a center. For what concerns double clusters, we analyzed only the brighter of the two subunits. The total number of objects surviving our selection procedures is 59. About half of our objects are local, $0.03<z<0.1$, while the other half are located at intermediate redshifts, $0.1<z<0.25$.

Although not complete, we have reason to believe that our sample is fairly representative of the cluster population as a whole. Indeed objects at $z>0.1$ were extracted by applying a redshift cut at $z=0.25$ from the sample analyzed in Leccardi \& Molendi (2008a,b), which we showed to be unaffected by substantial biases (Leccardi \& Molendi 2008b). Similarly the low-redshift half of our subsample is extracted from the Edge et al. (1990) flux limited sample by applying neutral cuts such as throwing away observations that are badly affected by soft proton flares. The only selection introducing a certain amount of bias was the one against extremely disturbed clusters for which it was impossible to define a center. We note that only 4 systems were excluded in this manner and that they will be reintroduced in a subsequent paper (Rossetti \& Molendi 2010), where we will make use of the 2D information provided by X-ray maps.

\section{Data analysis}

In this section we provide some information on the data analysis, a more thorough description may be found in Leccardi \& Molendi (2008a) and Leccardi \& Molendi (2008b). Here we recall some general issues and highlight a few differences between the analysis performed in this paper and the one conducted in our previous works.

\footnotetext{
1 http://xmm.esac.esa.int/external/xmm_news/items/ MOS1-CCD6/index. shtml

2 http://nedwww.ipac.caltech.edu
}

\subsection{Event file production}

Observation data files (ODF) were retrieved from the XMM-Newton archive and were subject to standard processing with the Science Analysis System (SAS) v7.0. The soft-proton cleaning was performed using a double filtering process, first in a hard (10-12 keV) and then in a soft (2-5 keV) energy range. The event files were filtered according to PATTERN and FLAG criteria. Bright point-like sources were detected, using a procedure based on the SAS task edetect_chain, and excluded from the event file (see Leccardi \& Molendi 2008b for details).

The $R_{\mathrm{SB}}$ indicator, i.e. the ratio between surface-brightness calculated inside and outside the field of view (see Eq. (1) in Leccardi \& Molendi 2008b), allowed us to exclude extremely polluted observations and to quantify the quiescent soft-proton (QSP) component surviving the double filtering process. Since local clusters fill the whole EPIC field of view, in contrast to what we did in Leccardi \& Molendi $(2008 \mathrm{~b})$, we measured $R_{\mathrm{SB}}$ above $9 \mathrm{keV}$ where EPIC effective areas rapidly decrease. We excluded all clusters for which the mean $R_{\mathrm{SB}}$ for the two MOS is greater than 2.0. The threshold is higher than chosen in Leccardi \& Molendi (2008b) because the aim of this work is to analyze the central regions, where the sensitivity to background variations is not as high as in the outskirts.

\subsection{Spectral analysis}

To investigate cluster properties in the central regions, we accumulated spectra in two regions: the inner region is a circle of radius $0.05 R_{180}$ and the outer region an annulus with bounding radii $0.05-0.20 R_{180}$ (the outer radius of the latter region is limited by the apparent size of the nearest clusters). Both IN and OUT regions are centered on the X-ray emission peak. We recall that $R_{180}$ is the radius within which the mean density is 180 times the critical density and that it was computed as in Leccardi \& Molendi (2008b). Since, as discussed in Sect. $4, R_{180}$ is itself computed from the temperature measured in the OUT region, we iterated the process until it converged to stable values of $R_{180}$. The first guess for $R_{180}$ was computed using temperatures from Leccardi \& Molendi (2008b) and the literature.

For each EPIC instrument and each region, we generated an effective area (ARF), and for each observation we generated redistribution functions (RMF) for MOS1, MOS2, and pn. Spectra accumulated in the central regions of clusters almost always have high statistical quality; therefore, the complicated procedures we developed for dealing with the background in the outer regions (see Appendices of Leccardi \& Molendi 2008b) are not strictly necessary and also EPIC pn data were used. For all three detectors (namely MOS1, MOS2, and pn), channels were assembled to have at least 25 counts for each group, as commonly done when using the $\chi^{2}$ statistic. We merged nine blank-field observations to accumulate background spectra. For each cluster observation, we calculated the count rate ratio, $Q$, between source and background observations above $9 \mathrm{keV}$ in an external ring $\left(10^{\prime}-12^{\prime}\right)$ of the field of view. We scaled the background spectrum by $Q$ and, for each region, subtracted it from the corresponding spectrum from cluster observation. This rough rescaling accounts for possible temporal variations of the instrumental background dominating at high energies without introducing substantial distortions to the source spectrum in the soft energy band where cosmic background components are more important and the source outshines the background by more than one order of magnitude. 
A. Leccardi et al.: Thermo-dynamic and chemical properties of the ICM

Table 1. Physical properties and observation details for the 59 clusters of our sample.

\begin{tabular}{|c|c|c|c|c|c|}
\hline Name & $z^{a}$ & $k T_{\text {OUT }^{b}}^{b}$ & Exp. time ${ }^{c}$ & $R_{\mathrm{SB}}^{d}$ & Filter \\
\hline Abell 4038 & 0.0300 & 3.0 & 78.1 & 1.45 & MEDIUM \\
\hline Abell 2199 & 0.0301 & 4.1 & 38.4 & 1.14 & THIN1 \\
\hline 2A $0335+096$ & 0.0349 & 3.6 & 230.8 & 1.02 & THIN1 \\
\hline Abell 2052 & 0.0355 & 2.9 & 85.1 & 0.99 & THIN1 \\
\hline Abell 576 & 0.0390 & 3.8 & 42.7 & 1.54 & MEDIUM \\
\hline Abell 3571 & 0.0391 & 6.3 & 43.5 & 1.54 & MEDIUM \\
\hline Abell 119 & 0.0442 & 6.0 & 54.1 & 1.62 & THIN1 \\
\hline MKW 03 & 0.0450 & 3.3 & 99.2 & 1.08 & THIN1 \\
\hline Abell 3376 & 0.0456 & 3.9 & 56.4 & 1.10 & MEDIUM \\
\hline Abell 1644 & 0.0470 & 4.2 & 42.0 & 1.40 & THIN1 \\
\hline Abell 4059 & 0.0475 & 4.0 & 64.9 & 1.11 & THIN1 \\
\hline Abell 3558 & 0.0480 & 5.2 & 126.4 & 1.00 & THICK \\
\hline Abell 3562 & 0.0480 & 4.3 & 116.6 & 1.85 & THIN1 \\
\hline Triangulum Austr. & 0.0510 & 9.2 & 27.3 & 1.15 & MEDIUM \\
\hline Hydra A & 0.0538 & 3.4 & 52.2 & 1.59 & THIN1 \\
\hline Abell 754 & 0.0542 & 8.7 & 30.8 & 1.11 & MEDIUM \\
\hline Abell 85 & 0.0551 & 5.5 & 34.6 & 1.04 & MEDIUM \\
\hline Abell 2319 & 0.0557 & 9.2 & 44.6 & 1.80 & MEDIUM \\
\hline Abell 3158 & 0.0597 & 4.9 & 54.0 & 1.58 & THIN1 \\
\hline Abell 1795 & 0.0625 & 5.4 & 97.3 & 1.29 & THIN1 \\
\hline Abell 399 & 0.0720 & 6.0 & 27.9 & 1.84 & THIN1 \\
\hline Abell 401 & 0.0740 & 7.3 & 34.7 & 1.76 & MEDIUM \\
\hline Abell 3112 & 0.0750 & 4.3 & 64.6 & 1.23 & MEDIUM \\
\hline Abell 2029 & 0.0773 & 6.2 & 30.8 & 1.17 & THIN1 \\
\hline Abell 2255 & 0.0806 & 6.2 & 25.1 & 1.37 & THIN1 \\
\hline Abell 1650 & 0.0838 & 5.4 & 75.0 & 1.29 & MEDIUM \\
\hline Abell 2597 & 0.0852 & 3.5 & 144.3 & 1.07 & THIN1 \\
\hline Abell S0084 & 0.1080 & 3.3 & 44.8 & 1.28 & THIN1 \\
\hline Abell 2034 & 0.1130 & 7.0 & 27.9 & 1.16 & THIN1 \\
\hline Abell 2051 & 0.1150 & 3.8 & 83.6 & 1.08 & THIN1 \\
\hline Abell 3814 & 0.1179 & 3.3 & 71.2 & 1.11 & THIN1 \\
\hline Abell 2050 & 0.1183 & 5.3 & 77.3 & 1.13 & THIN1 \\
\hline RXCJ1141.4-1216 & 0.1195 & 3.8 & 82.0 & 1.03 & THIN1 \\
\hline Abell 1084 & 0.1323 & 3.9 & 72.4 & 1.03 & THIN1 \\
\hline Abell 1068 & 0.1375 & 4.5 & 56.3 & 1.09 & MEDIUM \\
\hline Abell 3856 & 0.1379 & 6.4 & 54.6 & 1.11 & THIN1 \\
\hline Abell 3378 & 0.1410 & 4.9 & 58.3 & 1.07 & THIN1 \\
\hline Abell 0022 & 0.1424 & 5.7 & 41.9 & 1.02 & THIN1 \\
\hline Abell 1413 & 0.1427 & 6.7 & 71.7 & 1.10 & THIN1 \\
\hline Abell 2328 & 0.1470 & 5.6 & 71.1 & 1.07 & THIN1 \\
\hline Abell 3364 & 0.1483 & 6.7 & 67.1 & 1.12 & THIN1 \\
\hline Abell 2204 & 0.1522 & 8.5 & 51.2 & 1.06 & MEDIUM \\
\hline Abell 0907 & 0.1527 & 6.1 & 22.9 & 1.16 & THIN1 \\
\hline Abell 3888 & 0.1529 & 8.6 & 42.8 & 1.31 & THIN1 \\
\hline RXCJ2014.8-2430 & 0.1612 & 7.1 & 64.8 & 1.05 & THIN1 \\
\hline Abell 3404 & 0.1670 & 7.1 & 59.2 & 1.11 & THIN1 \\
\hline Abell 1914 & 0.1712 & 8.7 & 62.9 & 1.17 & THIN1 \\
\hline Abell 2218 & 0.1756 & 6.5 & 117.0 & 1.17 & THIN1 \\
\hline Abell 1689 & 0.1832 & 9.2 & 106.7 & 1.14 & THIN1 \\
\hline Abell 383 & 0.1871 & 4.4 & 82.3 & 1.33 & MEDIUM \\
\hline Abell 115N & 0.1971 & 5.1 & 103.2 & 1.20 & MEDIUM \\
\hline Abell 2163 & 0.2030 & 15.5 & 29.2 & 1.07 & THIN1 \\
\hline Abell 963 & 0.2060 & 6.5 & 69.4 & 1.19 & MEDIUM \\
\hline Abell 209 & 0.2060 & 6.6 & 49.3 & 1.19 & MEDIUM \\
\hline Abell 773 & 0.2170 & 7.5 & 45.6 & 1.16 & MEDIUM \\
\hline Abell 1763 & 0.2230 & 7.2 & 36.3 & 1.08 & MEDIUM \\
\hline Abell 2390 & 0.2280 & 11.2 & 29.4 & 1.11 & THIN1 \\
\hline Abell 2667 & 0.2300 & 7.7 & 59.9 & 1.48 & MEDIUM \\
\hline RX J2129.3+0005 & 0.2350 & 5.5 & 102.0 & 1.21 & MEDIUM \\
\hline
\end{tabular}

${ }^{(a)}$ Redshift taken from the NASA Extragalactic Database; ${ }^{(b)}$ reference temperature in keV derived from our analysis (see Sect. 3.2); ${ }^{(c)}$ total good exposure time in ks; ${ }^{(d)}$ intensity of residual soft protons (see Sect. 3.1). 


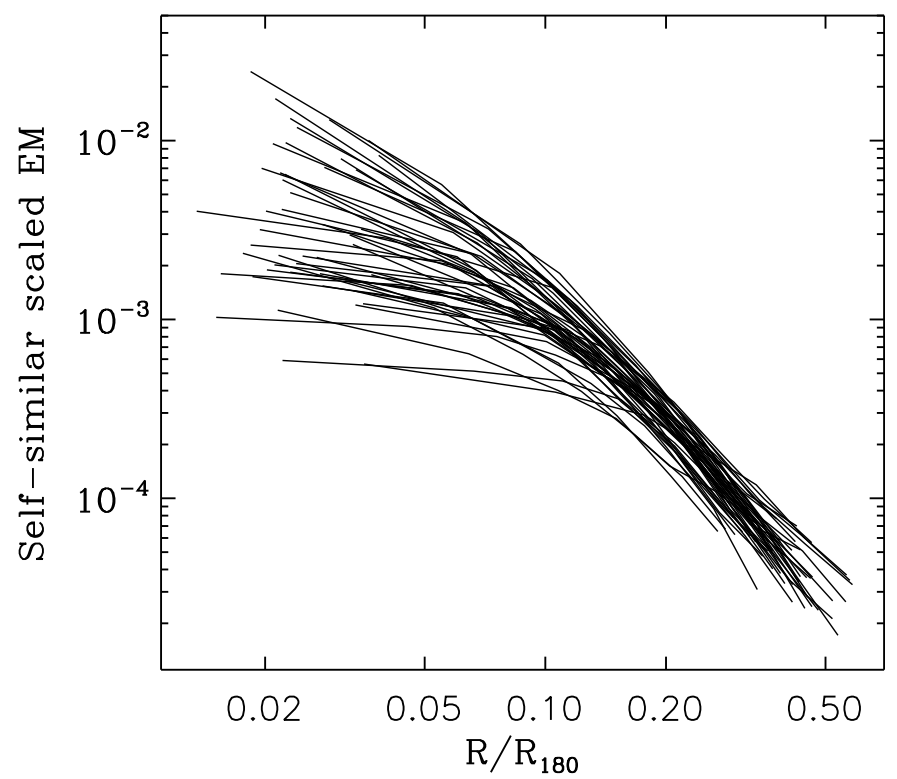

Fig. 1. Self-similar scaled emission measure profiles for the intermediate-redshift sample presented in Leccardi \& Molendi (2008a,b). The radius is in units of $R_{180}$ and the emission measure is scaled by $E M_{\text {SSS }}$ (see Eq. (A.1)).

The spectral fitting was performed in the $0.5-10.0 \mathrm{keV}$ energy band using the $\chi^{2}$ statistic, with an absorbed thermal model (WABS*MEKAL in XSPEC v11.3 ${ }^{3}$ ). We fit spectra leaving temperature and normalization free to vary. The metallicity ${ }^{4}$ was constrained between $\pm 5 Z_{\odot}$ (see the discussion in Appendix A of Leccardi \& Molendi 2008a). The redshift was constrained between $\pm 5 \%$ of the optical measurement, and the equivalent hydrogen column density along the line of sight, $N_{\mathrm{H}}$, was fixed to the $21 \mathrm{~cm}$ measurement (Dickey \& Lockman 1990). Finally, for each quantity we computed the average over the three (MOS1, MOS2, pn) values and derived the projected emission measure, $E M$, as the ratio between the normalization and the area of the region expressed in square arcminutes.

\section{Defining interesting quantities}

To characterize temperature and emission measure gradients, we need to compare the central to a global value for such quantities; however, for local clusters it was only possible to perform reliable measurements out to a small $(\approx 20 \%)$ fraction of $R_{180}$. As a temperature reference, we used $k T_{\text {OUT }}$ (see Sect. 3.2), which is found to be a good proxy for the global temperature from Fig. 4 of Leccardi \& Molendi (2008b). A reference value for the emission measure should be measured at $\approx 0.4 R_{180}$, where profiles show a remarkable degree of similarity (see Fig. 1). We considered two different proxies for the emission measure at large radii finding that one of them, namely the emission measure calculated in the outer region, $E M_{\mathrm{OUT}}$, does somewhat better than the other. We adopted $E M_{\text {OUT }}$ as a proxy for the emission measure at large radii. The interested reader may find a detailed analysis of how well $E M_{0}$ and the other proxy approximate the emission measure at large radii in Appendix A. For the less technically minded readers, suffice it to say that $E M_{0}$ reproduces the emission measure around $0.4 R_{180}$ to better than $20 \%$.

\footnotetext{
${ }^{3}$ http://heasarc.nasa.gov/docs/xanadu/xspec/xspec11/ index.html

${ }^{4}$ The solar abundances were taken from Anders \& Grevesse (1989).
}

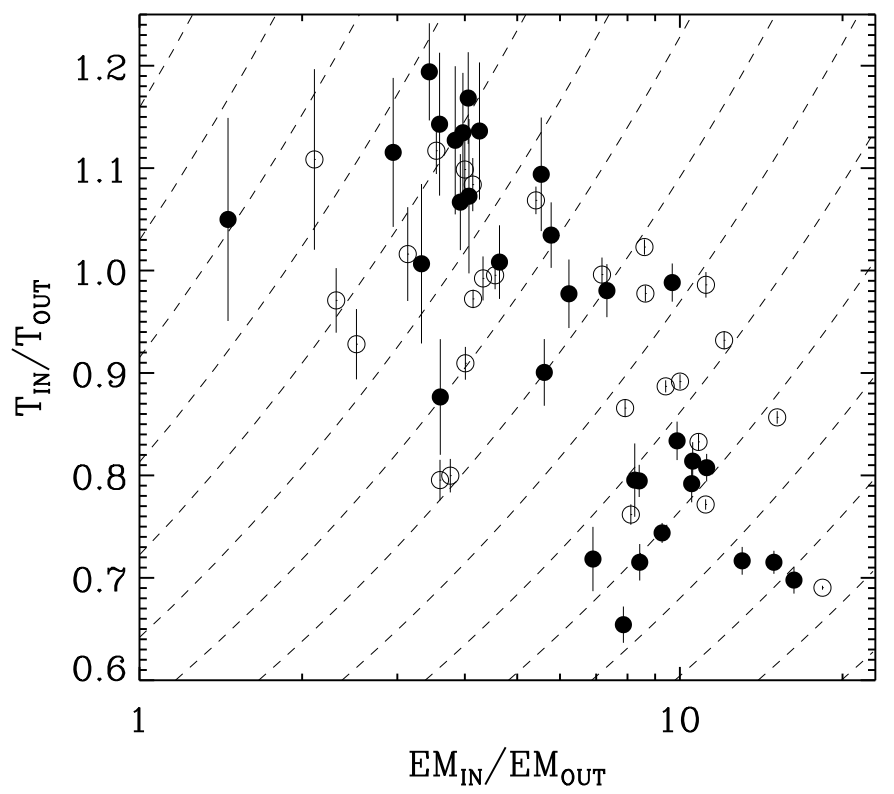

Fig. 2. Comparison of temperature and emission measure ratios for all clusters. The dashed curves indicate the regions of the plot where the pseudo-entropy ratio, $\sigma$, defined in Eq. (1), is constant. Clusters with the strongest pseudo-entropy variations populate the bottom-right corner. Open and filled circles indicate local (i.e. $z<0.1$ ) and distant (i.e. $z>$ $0.1)$ clusters, respectively.

In Fig. 2 we compare the temperature ratio, $T_{\mathrm{IN}} / T_{\mathrm{OUT}}$, with the emission measure ratio, $E M_{\mathrm{IN}} / E M_{\mathrm{OUT}}$. As expected, there is a clear, but quite scattered, correlation: more precisely, the stronger the emission measure peak, the stronger the temperature drop. A key thermo-dynamic observable in describing clusters is the entropy (e.g. Ponman et al. 2003; Voit 2005; Pratt et al. 2006), which is commonly defined as $S \equiv T_{\mathrm{X}} \times n_{\mathrm{e}}^{-2 / 3}$, where $T_{\mathrm{X}}$ and $n_{\mathrm{e}}$ are the deprojected temperature and electron density. In the literature (e.g. Rossetti et al. 2007), it is common to define a pseudo-entropy from projected quantities as $s \equiv T \times E M^{-1 / 3}$. Since we are interested in comparing core with cluster properties, we use our emission measure and temperature ratios to define a pseudo-entropy ratio,

$\sigma \equiv\left(T_{\mathrm{IN}} / T_{\mathrm{OUT}}\right) \times\left(E M_{\mathrm{IN}} / E M_{\mathrm{OUT}}\right)^{-1 / 3}$.

The pseudo-entropy ratio has been found to be well-correlated with the entropy ratio, which is computed using deprojected quantities (see Rossetti \& Molendi 2010, for a detailed comparison). In Fig. 2 the dashed curves indicate the regions where $\sigma$ is constant; clusters with the strongest variations (i.e. lower ratios) of pseudo-entropy fill the bottom-right corner and are usually known as cool core clusters (see Sects. 5 and 6 for a detailed discussion). Values of the pseudo-entropy ratio for all objects in our sample are reported in Table 2.

The central cooling time is another quantity widely used in the literature (e.g. Peres et al. 1998) to estimate the degree of relaxation of clusters. As for the entropy, we defined a pseudocooling-time, $t_{\mathrm{pc}} \equiv T^{1 / 2} \times E M^{-1 / 2}$, and a pseudo-cooling-time ratio,

$\tau \equiv\left(T_{\mathrm{IN}} / T_{\mathrm{OUT}}\right)^{1 / 2} \times\left(E M_{\mathrm{IN}} / E M_{\mathrm{OUT}}\right)^{-1 / 2}$.

When ordering clusters according to $\tau$, we find essentially the same results as when using $\sigma$.

An interesting property of our pseudo-entropy ratio is that it can be constructed from data of moderate statistical quality such 
A. Leccardi et al.: Thermo-dynamic and chemical properties of the ICM

Table 2. Pseudo-entropy ratio and metal abundances for the inner region of the 59 objects in our sample.

\begin{tabular}{|c|c|c|c|}
\hline Name & $\sigma^{a}$ & $\begin{array}{l}\text { Entropy } \\
\text { Classif. }^{b}\end{array}$ & $Z_{\mathrm{IN}} / Z_{\odot}^{c}$ \\
\hline Abell 4038 & $0.499 \pm 0.004$ & MEC & $0.41 \pm 0.01$ \\
\hline Abell 2199 & $0.414 \pm 0.003$ & LEC & $0.45 \pm 0.01$ \\
\hline 2A $0335+096$ & $0.262 \pm 0.001$ & LEC & $0.66 \pm 0.00$ \\
\hline Abell 2052 & $0.420 \pm 0.003$ & LEC & $0.55 \pm 0.01$ \\
\hline Abell 576 & $0.609 \pm 0.013$ & MEC & $0.53 \pm 0.04$ \\
\hline Abell 3571 & $0.608 \pm 0.008$ & MEC & $0.37 \pm 0.02$ \\
\hline Abell 119 & $0.734 \pm 0.024$ & HEC & $0.28 \pm 0.05$ \\
\hline MKW 03 & $0.477 \pm 0.004$ & MEC & $0.52 \pm 0.01$ \\
\hline Abell 3376 & $0.682 \pm 0.025$ & HEC & $0.30 \pm 0.05$ \\
\hline Abell 1644 & $0.519 \pm 0.013$ & MEC & $0.74 \pm 0.05$ \\
\hline Abell 4059 & $0.434 \pm 0.004$ & LEC & $0.60 \pm 0.02$ \\
\hline Abell 3558 & $0.605 \pm 0.006$ & MEC & $0.38 \pm 0.01$ \\
\hline Abell 3562 & $0.601 \pm 0.008$ & MEC & $0.50 \pm 0.02$ \\
\hline Triangulum Austr. & $0.675 \pm 0.016$ & HEC & $0.29 \pm 0.03$ \\
\hline Hydra A & $0.406 \pm 0.004$ & LEC & $0.34 \pm 0.01$ \\
\hline Abel & $0.514 \pm 0$ & MEC & $0.31 \pm 0.03$ \\
\hline Abell 85 & $0.379 \pm 0.005$ & LEC & $0.51 \pm 0.02$ \\
\hline Abell 2319 & $0.572 \pm 0$ & MEC & $0.26 \pm 0.02$ \\
\hline Abell 3158 & $0.733 \pm 0.015$ & HEC & $0.32 \pm 0.03$ \\
\hline Abell 1795 & $0.345 \pm 0.002$ & LEC & $0.40 \pm 0.01$ \\
\hline Abell 399 & $0.694 \pm 0.031$ & HEC & $0.27 \pm 0.06$ \\
\hline Abell 401 & $0.692 \pm 0.021$ & HEC & $0.25 \pm 0.03$ \\
\hline Abell 3112 & $0.376 \pm 0.004$ & LEC & $0.59 \pm 0.02$ \\
\hline Abell 2029 & $0.441 \pm 0.006$ & LEC & $0.47 \pm 0.02$ \\
\hline Abell 2255 & $0.865 \pm 0.069$ & HEC & $0.17 \pm 0.12$ \\
\hline Abell 1650 & $0.516 \pm 0.009$ & MEC & $0.40 \pm 0.02$ \\
\hline Abell 2597 & $0.346 \pm 0.002$ & LEC & $0.36 \pm 0.01$ \\
\hline Abell S0084 & $618 \pm 0.031$ & MEC & $0.71 \pm 0.10$ \\
\hline Abell 2034 & $.778 \pm 0.0$ & $\mathrm{EC}$ & $0.47 \pm 0.11$ \\
\hline & $16 \pm$ & IEC & $0.27 \pm 0.10$ \\
\hline Abel & $0.371 \pm$ & LEC & $0.66 \pm 0.04$ \\
\hline Abell 2050 & $0.733 \pm 0.028$ & HEC & $0.32 \pm 0.05$ \\
\hline RXCJ1141.4-1216 & $0.354 \pm 0.005$ & LEC & $0.64 \pm 0.03$ \\
\hline Abell 1084 & $0.361 \pm 0.006$ & LEC & $0.42 \pm 0.02$ \\
\hline & $0.304 \pm 0.006$ & LEC & $0.48 \pm 0.02$ \\
\hline Abell 3856 & $0.605 \pm 0.022$ & MEC & $0.34 \pm 0.05$ \\
\hline Abell 3378 & $0.389 \pm 0.009$ & LEC & $0.56 \pm 0.03$ \\
\hline Abell 0022 & $0.701 \pm 0.041$ & HEC & $0.31 \pm 0.08$ \\
\hline Abell 1413 & $0.505 \pm 0.013$ & MEC & $0.33 \pm 0.03$ \\
\hline Abell 2328 & $0.926 \pm 0.087$ & HEC & $0.35 \pm 0.18$ \\
\hline Abell 3364 & $0.676 \pm 0.030$ & HEC & $0.35 \pm 0.06$ \\
\hline Abell 2204 & $0.290 \pm 0.005$ & LEC & $0.51 \pm 0.02$ \\
\hline Abell 0907 & $0.394 \pm 0.018$ & LEC & $0.46 \pm 0.07$ \\
\hline Abel & $.717 \pm 0.037$ & HEC & $0.26 \pm 0.07$ \\
\hline RXCJ2014.8-2430 & $0.275 \pm 0.005$ & LEC & $0.45 \pm 0.02$ \\
\hline Abell 3404 & $0.507 \pm 0.018$ & MEC & $0.30 \pm 0.05$ \\
\hline Abell 1914 & $0.577 \pm 0.018$ & MEC & $0.29 \pm 0.04$ \\
\hline Abell 2218 & $0.791 \pm 0.031$ & HEC & $0.28 \pm 0.06$ \\
\hline Abell 1689 & $0.464 \pm 0.009$ & MEC & $0.31 \pm 0.02$ \\
\hline Abell 383 & $0.361 \pm 0.008$ & LEC & $0.52 \pm 0.03$ \\
\hline Abell 115N & $0.329 \pm 0.009$ & LEC & $0.42 \pm 0.03$ \\
\hline Abell 2163 & $0.720 \pm 0.046$ & HEC & $0.34 \pm 0.10$ \\
\hline Abell 963 & $0.572 \pm 0.037$ & MEC & $0.51 \pm 0.10$ \\
\hline Abell 209 & $0.531 \pm 0.018$ & MEC & $0.36 \pm 0.05$ \\
\hline Abell 773 & $0.672 \pm 0.047$ & HEC & $0.30 \pm 0.10$ \\
\hline Abell 1763 & $0.674 \pm 0.052$ & HEC & $0.52 \pm 0.15$ \\
\hline Abell 2390 & $0.377 \pm 0.016$ & LEC & $0.42 \pm 0.05$ \\
\hline Abell 2667 & $0.351 \pm 0.009$ & LEC & $0.47 \pm 0.03$ \\
\hline RX J2129.3+0005 & $0.391 \pm 0.008$ & LEC & $0.35 \pm 0.03$ \\
\hline
\end{tabular}

(a) Pseudo-entropy ratio, see Sect. 4 for details; ${ }^{(b)}$ pseudo entropy class, see Sect. 6.1 for details; ${ }^{(c)}$ metal abundance in central region, see Sect. 7.2 for details. 
as serendipitous observations of clusters in deep XMM-Newton and Chandra observations. In Sect. 6.1 we employ $\sigma$ to divide clusters into 3 broad categories namely: low-entropy core (LEC), medium-entropy core (MEC), and high-entropy core (HEC) systems. Before we proceed with our entropy-based classification, we must first consider other classification schemes based, at least in part, on different cluster properties.

\section{Alternative classification schemes}

We wish to provide alternative classification schemes with which to compare our entropy-based scheme. More specifically we wish to compare it with 1) the traditional cool core/non-cool core classification; and 2) a classification scheme based on dynamical properties. To avoid circularity, we want the alternative classification schemes to be as independent as possible of our measurements. Therefore, for the cool core/non-cool core classification, we rely on how astronomers have classified these clusters in the literature and not on our own data. More specifically, we divide objects into 3 classes: cool cores (CC), intermediate systems (INT), and non-cool cores (NCC). We classify as CC those systems for which we find evidence in the literature of a temperature decrement and a peaked surface brightness profile. NCC are those that possess neither of the above properties, while INT systems are those that possess one or the other, or alternatively both, but not as well developed as in full blown cool core systems. We refrain from providing a more quantitative classification for two main reasons: 1) this would be rather difficult to derive from the literature, since different authors make use of somewhat different criteria and certainly do not analyze data in a homogenous fashion; 2) our own entropy classification, as we see in Sect. 6.1, is best understood as a more quantitative classification scheme along these very lines.

For the dynamical classification, we divide objects in 2 classes: mergers (MRG), and systems for which we do not find any evidence of a merger (NOM). We consider evidence for substantial cluster-wide interaction leading to a merger classification the following phenomena: 1) cluster-wide diffuse radio emission, such as radio haloes and relics; 2) multi-peaked velocity distribution from optical spectroscopy, or evidence of substructure from the combination of optical spectroscopy and photometry, or evidence for multiple mass peaks from lensing analysis; 3) significant irregularities observed in X-rays both in morphology and temperature maps. The lack of diffuse radio emission or of multi-peaked velocity distribution from optical spectroscopy is in itself insufficient for classifying an object as relaxed. Similarly the absence of significant substructure on cluster-wide scales in X-ray images is not in itself proof that substructure does not exist or that the system under scrutiny is relaxed. For these reasons it is rather difficult to classify a system as relaxed, what we can ascertain is that some systems lack evidence of merger activity. Consequently we classify all objects for which we do not have evidence of merging as "no observed merging" (NOM) systems. We reiterate that an NOM system is not necessarily a relaxed system but rather a system for which we do not have evidence of merging activity, in the sense described previously in this paragraph.

In Table 3 we provide results from our classification work. Columns 2 and 3 refer to radio emission, in Col. 2 we indicate clusters with radio haloes by " $H$ ", clusters without radio haloes by "N", clusters with tentative radio haloes by "?", clusters with mini-radio haloes by " $\mathrm{M}$ " and clusters with radio relics by " $\mathrm{R}$ ". Column 3 provides references for Col. 2. Columns 4 and 5 refer to optical emission, we indicate clusters with substructure in the forms described above by "Y", clusters without by " $N$ " and uncertain cases by "?". Column 5 provides references for Col. 4. Columns 6 and 7 refer to X-ray emission, in Col. 6 we indicate clusters that have been identified as cool core (or cooling flow) systems by "CC"; clusters that have been identified as non-cool core (or non-cooling flow) systems by "NCC"; clusters with intermediate cores by "INT" and clusters identified as mergers by "MRG". Column 7 provides references for Col. 6. In Col. 9 we provide our core based classification and in Col. 10 our dynamical classification. For all objects where a classification cannot be desumed directly from information in Cols. 2 to 7 , we provide a note explaining how the classification was derived. In Col. 8 we indicate those objects for which we provide a note with "Y" and those for which we do not with "N".

For the core classification, we classify 24 systems as CC, 25 as NCC and 10 as INT. For the dynamical classification we classify 19 objects as MRG and 40 as NOM.

\subsection{Notes on individual objects}

\subsubsection{A4038}

\section{Core classification}

Analysis of Chandra data provides a central cooling time of $1.3 \mathrm{Gyr}$ (Sun et al. 2007) and a flat temperature profile (Sanderson et al. 2006); moreover, inspection of Chandra and $X M M-N e w t o n$ images show evidence of an irregular core. This system clearly does not host a full blown cool core, so we classify it as intermediate.

\section{Dynamical classification}

Diffuse radio emission has been reported for this source (Slee \& Roy 1998; Slee et al. 2001), although the emission is located at the center of the cluster. Its appearance is more similar to that of a radio relic than to a radio halo, and it is most likely a remnant associated to the radio galaxy observed in this system. Optical observations (Burgett et al. 2004) provide controversial evidence of substructure on large scales. The evidence pointing to a merger is in our opinion insufficient, so we chose to classify this object as NOM.

\subsubsection{A3571}

\section{Core classification}

Analysis of Chandra data provides a central cooling time of 1.3 Gyr (Sun et al. 2007); however there is no evidence of a temperature decrement in the core (Sakelliou \& Ponman 2006). This system does not host a full blown cool core so we classify it as INT.

\section{Dynamical classification}

On the basis of the multi-wavelength properties of the A3571 cluster complex, Venturi et al. (2002) propose that A3571 is a very advanced merger and explain the radio properties derived from their study in the light of this hypothesis. We deem the evidence collected by Venturi et al. (2002) insufficient for classifying A3571 as a merger and conservatively catalog it as NOM.

\subsubsection{A1650}

\section{Core classification}

Analysis of Chandra data, readily available through the ACCEPT archive (Cavagnolo et al. 2009), show this object to be of an intermediate nature possessing many of the traits typical of cool cores, such as an abundance excess and a temperature drop, albeit with a relatively high core entropy $\simeq 40 \mathrm{keV} \mathrm{cm}^{2}$. 
A. Leccardi et al.: Thermo-dynamic and chemical properties of the ICM

Table 3. Classification of objects based on radio, optical and X-ray properties.

\begin{tabular}{|c|c|c|c|c|c|c|c|c|c|}
\hline Name & $R^{a}$ & R. ref. & $\mathrm{O}^{b}$ & O. ref. & $\mathrm{X}$-ray ${ }^{c}$ & X-ray ref. & Note & Core $\mathrm{Cl}$. & Dyn. Cl. \\
\hline Abell 4038 & $\mathrm{H}$ & S198, S101 & $?$ & $\mathrm{Bu} 04$ & $\mathrm{NCC}$ & Sn06 & $\mathrm{Y}$ & INT & NOM \\
\hline Abell 2199 & - & & $?$ & $\mathrm{Oe} 01$ & $\mathrm{CC}$ & Jo02 & $\mathrm{N}$ & $\mathrm{CC}$ & NOM \\
\hline 2A $0335+096$ & - & & - & & $\mathrm{CC}$ & $\mathrm{Ma03}$ & $\mathrm{N}$ & $\mathrm{CC}$ & NOM \\
\hline Abell 2052 & - & & $\mathrm{N}$ & Di87, Ma92 & $\mathrm{CC}$ & $\mathrm{B} 103$ & $\mathrm{~N}$ & $\mathrm{CC}$ & NOM \\
\hline Abell 576 & $\mathrm{~N}$ & $\mathrm{Fe} 07$ & - & & MRG \& NCC & Mo96, Ke04, Du07 & $\mathrm{N}$ & $\mathrm{NCC}$ & MRG \\
\hline Abell 3571 & $\mathrm{~N}$ & $\mathrm{Ve} 02$ & - & & NCC & Oh06, Sn06, Da08 & $\mathrm{Y}$ & INT & NOM \\
\hline Abell 119 & $\mathrm{~N}$ & $\mathrm{Mu} 04$ & - & & MRG \& NCC & Sr06, Ro06 & $\mathrm{N}$ & $\mathrm{NCC}$ & MRG \\
\hline MKW 03 & - & & - & & $\mathrm{CC}$ & $\mathrm{Ma} 02, \mathrm{Su} 07$ & $\mathrm{~N}$ & $\mathrm{CC}$ & NOM \\
\hline Abell 3376 & $\mathrm{R}$ & $\mathrm{Ba} 06$ & $\mathrm{~N}$ & Gi97 & MRG \& NCC & Ro06, Ca09 & $\mathrm{N}$ & $\mathrm{NCC}$ & MRG \\
\hline Abell 1644 & - & & $\mathrm{N}$ & Tu01 & INT & Re04, Du05 & $\mathrm{N}$ & INT & NOM \\
\hline Abell 4059 & - & & - & & $\mathrm{CC}$ & Ch04, Ca09 & $\mathrm{N}$ & $\mathrm{CC}$ & NOM \\
\hline Abell 3558 & $\mathrm{~N}$ & Ba98 & $\mathrm{Y}$ & Ba98 & INT & Ro07 & $\mathrm{N}$ & INT & NOM \\
\hline Abell 3562 & $\mathrm{H}$ & Gi05 & $\mathrm{Y}$ & $\mathrm{Ba} 98$ & MRG \& NCC & Fi04 & $\mathrm{N}$ & $\mathrm{NCC}$ & MRG \\
\hline Triangulum Austr. & - & & - & & NCC & $\mathrm{Ch07}$ & $\mathrm{N}$ & $\mathrm{NCC}$ & NOM \\
\hline Hydra A & - & & - & & $\mathrm{CC}$ & Da01 & $\mathrm{N}$ & $\mathrm{CC}$ & NOM \\
\hline Abell 754 & $\mathrm{H}$ & $\mathrm{Ka} 01, \mathrm{Ba} 03$ & $?$ & Ro98 & MRG \& NCC & $\mathrm{Kr} 03, \mathrm{He} 04$ & $\mathrm{~N}$ & $\mathrm{NCC}$ & MRG \\
\hline Abell 85 & - & & $\mathrm{N}$ & Ma92, Br09 & MRG \& CC & $\mathrm{Ke} 02$, Dr05 & $\mathrm{N}$ & $\mathrm{CC}$ & MRG \\
\hline Abell 2319 & $\mathrm{H}$ & $\mathrm{Fe} 97, \mathrm{Ca} 06$ & $\mathrm{Y}$ & Oe95 & MRG \& NCC & Oh04 & $\mathrm{N}$ & $\mathrm{NCC}$ & MRG \\
\hline Abell 3158 & - & & Y & Jo08 & MRG \& NCC & Sn08, Gh09 & $\mathrm{N}$ & $\mathrm{NCC}$ & MRG \\
\hline Abell 1795 & - & & - & & $\mathrm{CC}$ & Et02 & $\mathrm{N}$ & $\mathrm{CC}$ & NOM \\
\hline Abell 399 & $\mathrm{~N}$ & Gi06 & $\mathrm{Y}$ & Gi97 & MRG \& NCC & $\mathrm{Sa} 04, \mathrm{Ro} 06$ & $\mathrm{~N}$ & $\mathrm{NCC}$ & MRG \\
\hline Abell 401 & $\mathrm{H}$ & $\mathrm{Ba} 03$ & Y & Gi97 & MRG \& NCC & $\mathrm{Sa} 04, \mathrm{Bo} 08$ & $\mathrm{~N}$ & $\mathrm{NCC}$ & MRG \\
\hline Abell 3112 & - & & - & & $\mathrm{CC}$ & $\mathrm{Ta} 03$ & $\mathrm{~N}$ & $\mathrm{CC}$ & NOM \\
\hline Abell 2029 & - & & - & & $\mathrm{CC}$ & $\mathrm{Cl04}$ & $\mathrm{N}$ & $\mathrm{CC}$ & NOM \\
\hline Abell 2255 & $\mathrm{H}$ & Pi08 & - & & MRG \& NCC & $\mathrm{Sa06}$ & $\mathrm{N}$ & $\mathrm{NCC}$ & MRG \\
\hline Abell 1650 & - & & - & & $\mathrm{CC}$ & Do05, Ca09 & $\mathrm{Y}$ & INT & NOM \\
\hline Abell 2597 & - & & - & & $\mathrm{CC}$ & $\mathrm{Mo05}, \mathrm{Ca} 09$ & $\mathrm{~N}$ & $\mathrm{CC}$ & NOM \\
\hline Abell S0084 & - & & - & & INT & Si09 & $\mathrm{N}$ & INT & $\mathrm{NOM}$ \\
\hline Abell 2034 & - & & - & & MRG \& NCC & $\mathrm{Ke} 03, \mathrm{~B} 107$ & $\mathrm{~N}$ & $\mathrm{NCC}$ & MRG \\
\hline Abell 2051 & - & & - & & NCC & Pr07, Cr08 & $\mathrm{N}$ & $\mathrm{NCC}$ & NOM \\
\hline Abell 3814 & - & & - & & $\mathrm{CC}$ & Cr08, Le08 & $\mathrm{N}$ & $\mathrm{CC}$ & NOM \\
\hline Abell 2050 & - & & - & & $\mathrm{NCC}$ & Pr07, Cr08 & $\mathrm{N}$ & $\mathrm{NCC}$ & NOM \\
\hline RXCJ1141.4-1216 & - & & - & & $\mathrm{CC}$ & Pr07, Cr08 & $\mathrm{N}$ & $\mathrm{CC}$ & NOM \\
\hline Abell 1084 & - & & - & & $\mathrm{CC}$ & Pr07, Cr08 & $\mathrm{N}$ & $\mathrm{CC}$ & NOM \\
\hline Abell 1068 & - & & - & & $\mathrm{CC}$ & Wi04 & $\mathrm{N}$ & $\mathrm{CC}$ & NOM \\
\hline Abell 3856 & - & & - & & $\mathrm{NCC}$ & Pr07, Cr08 & $\mathrm{N}$ & $\mathrm{NCC}$ & NOM \\
\hline Abell 3378 & - & & - & & $\mathrm{CC}$ & Pr07, Cr08 & $\mathrm{N}$ & $\mathrm{CC}$ & NOM \\
\hline Abell 0022 & - & & - & & $\mathrm{NCC}$ & Pr07, Cr08 & $\mathrm{N}$ & $\mathrm{NCC}$ & NOM \\
\hline Abell 1413 & - & & - & & INT & Vi05, B107, Ca09 & $\mathrm{N}$ & INT & NOM \\
\hline Abell 2328 & - & & - & & NCC & Pr07, Cr08 & $\mathrm{N}$ & NCC & NOM \\
\hline Abell 3364 & - & & - & & $\mathrm{NCC}$ & Pr07, Cr08 & $\mathrm{N}$ & $\mathrm{NCC}$ & NOM \\
\hline Abell 2204 & - & & - & & $\mathrm{CC}$ & Sa09 & $\mathrm{N}$ & $\mathrm{CC}$ & NOM \\
\hline Abell 0907 & - & & - & & $\mathrm{CC}$ & Vi05, Cr08 & $\mathrm{N}$ & $\mathrm{CC}$ & NOM \\
\hline Abell 3888 & - & & - & & $\mathrm{NCC}$ & Cr08 & $\mathrm{N}$ & $\mathrm{NCC}$ & NOM \\
\hline RXCJ2014.8-2430 & - & & - & & $\mathrm{CC}$ & $\mathrm{Cr} 08$ & $\mathrm{~N}$ & $\mathrm{CC}$ & NOM \\
\hline Abell 3404 & - & & - & & INT & Cr08 & $\mathrm{N}$ & INT & NOM \\
\hline Abell 1914 & $\mathrm{H}$ & $\mathrm{Ba} 03$ & - & & MRG \& NCC & Go04, B107 & $\mathrm{N}$ & $\mathrm{NCC}$ & MRG \\
\hline Abell 2218 & $\mathrm{H}$ & Gi00 & $\mathrm{Y}$ & Gi97 & MRG \& NCC & Go04, B107 & $\mathrm{N}$ & NCC & MRG \\
\hline Abell 1689 & - & & $?$ & Gi01, Lo06 & MRG \& CC & $\mathrm{Pe} 98, \mathrm{An} 04, \mathrm{Ca} 09$ & $\mathrm{Y}$ & INT & NOM \\
\hline Abell 383 & - & & - & & $\mathrm{CC}$ & Vi05, Ca09 & $\mathrm{N}$ & $\mathrm{CC}$ & NOM \\
\hline Abell 115N & $?$ & Go01 & Y & $\mathrm{Ba} 07 \mathrm{~b}$ & MRG \& CC & Gu05 & $\mathrm{N}$ & $\mathrm{CC}$ & MRG \\
\hline Abell 2163 & $\mathrm{H}$ & $\mathrm{Fe} 01$ & $\mathrm{Y}$ & $\mathrm{Ma08}$ & MRG \& NCC & Go04 & $\mathrm{N}$ & $\mathrm{NCC}$ & MRG \\
\hline Abell 963 & $\mathrm{~N}$ & $\mathrm{Ca} 08$ & - & & NCC & 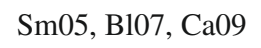 & $\mathrm{Y}$ & INT & NOM \\
\hline Abell 209 & $\mathrm{H}$ & $\mathrm{Ve} 07$ & $\mathrm{Y}$ & $\mathrm{Da} 02, \mathrm{Me} 04$ & $\mathrm{NCC}$ & $\mathrm{Ca} 09$ & $\mathrm{~N}$ & $\mathrm{NCC}$ & MRG \\
\hline Abell 773 & $\mathrm{H}$ & Go01 & $\mathrm{Y}$ & $\mathrm{Ba} 07 \mathrm{a}$ & MRG \& NCC & Go04, Ca09 & $\mathrm{N}$ & $\mathrm{NCC}$ & MRG \\
\hline
\end{tabular}


Table 3. continued.

\begin{tabular}{|c|c|c|c|c|c|c|c|c|c|}
\hline Name & $\mathrm{R}^{a}$ & R. ref. & $\mathrm{O}^{b}$ & O. ref. & X-ray ${ }^{c}$ & X-ray ref. & Note & Core $\mathrm{Cl}$. & Dyn. Cl. \\
\hline Abell 1763 & $\mathrm{~N}$ & $\mathrm{Ve} 08$ & $\mathrm{Y}$ & $\mathrm{Fa} 08$ & MRG \& NCC & Du08, Ca09 & $\mathrm{N}$ & $\mathrm{NCC}$ & MRG \\
\hline Abell 2390 & $\mathrm{M}$ & $\mathrm{Ba} 03$ & $\mathrm{~N}$ & Le91 & $\mathrm{CC}$ & Vi05, Ca09 & $\mathrm{N}$ & $\mathrm{CC}$ & $\mathrm{NOM}$ \\
\hline Abell 2667 & $\mathrm{~N}$ & Ve08 & - & & $\mathrm{CC}$ & $\mathrm{Co} 06, \mathrm{Ca} 09$ & $\mathrm{~N}$ & $\mathrm{CC}$ & $\mathrm{NOM}$ \\
\hline RX J2129.3+0005 & - & & - & & $\mathrm{CC}$ & Ca09 & $\mathrm{N}$ & $\mathrm{CC}$ & $\mathrm{NOM}$ \\
\hline
\end{tabular}

Notes. ${ }^{(a)}$ Radio emission; ${ }^{(b)}$ optical substructure; ${ }^{(c)}$ X-ray classification; a detailed description of the table is provided in Sect. 5. References. References divided by band of electromagnetic spectrum.

References for radio observations: (S198) Slee \& Roy (1998); (S101) Slee et al. (2001); (Fe07) Feretti \& Giovannini (2007); (Ve02) Venturi et al. (2002); (Mu04) Murgia et al. (2004); (Ba06) Bagchi et al. (2006); (Ba98) Bardelli et al. (1998); (Gi05) Giacintucci et al. (2005); (Ka01) Kassim et al. (2001); (Ba03) Bacchi et al. (2003); (Fe97) Feretti et al. (1997); (Ca06) Cassano et al. (2006); (Gi06) Giovannini et al. (2006); (Pi08) Pizzo et al. (2008); (Gi00) Giovannini \& Feretti (2000); (Go01) Govoni et al. (2001); (Fe01) Feretti et al. (2001); (Ca08) Cassano et al. (2008); (Ve07) Venturi et al. (2007); (Ve08) Venturi et al. (2008).

References for optical observations: (Bu04) Burgett et al. (2004); (Oe01) Oegerle \& Hill (2001); (Di87) Dixon et al. (1987); (Ma92) Malumuth et al. (1992); (Br09) Bravo-Alfaro et al. (2009); (Gi97) Girardi et al. (1997); (Tu01) Tustin et al. (2001); (Ro98) Roettiger et al. (1998); (Oe95) Oegerle et al. (1995); (Jo08) Johnston-Hollitt et al. (2008); (Gi01) Girardi \& Mezzetti (2001); (Lo06) Łokas et al. (2006); (Ba07b) Barrena et al. (2007b); (Ma08) Maurogordato et al. (2008); (Ba07a) Barrena et al. (2007a); (Fa08) Fadda et al. (2008); (Le91) Leborgne et al. (1991).

References for X-ray observations: (Sa06) Sakelliou \& Ponman (2006); (Jo02) Johnstone et al. (2002); (Ma03) Mazzotta et al. (2003); (B103) Blanton et al. (2003); (Mo96) Mohr et al. (1996); (Ke04) Kempner \& David (2004); (Du07) Dupke et al. (2007); (Oh06) O’Hara et al. (2006); (Sn06) Sanderson et al. (2006); (Da08) David \& Nulsen (2008); (Sr06) Sarazin (2006); (Ro06) Rossetti (2006); (Ma02) Mazzotta et al. (2002); (Su07) Sun et al. (2007); (Re04) Reiprich et al. (2004); (Du05) Dunn et al. (2005); (Ch04) Choi et al. (2004); (Ro07) Rossetti et al. (2007); (Fi04) Finoguenov et al. (2004); (Ch07) Chen et al. (2007); (Da01) David et al. (2001); (Kr03) Krivonos et al. (2003); (He04) Henry et al. (2004); (Ke02) Kempner et al. (2002); (Dr05) Durret et al. (2005); (Oh04) O'Hara et al. (2004); (Sn08) Snowden et al. (2008); (Gh09) Ghizzardi et al. (2009); (Et02) Ettori et al. (2002); (Sa04) Sakelliou \& Ponman (2004); (Bo08) Bourdin \& Mazzotta (2008); (Ta03) Takizawa et al. (2003); (Cl04) Clarke et al. (2004); (Sa06) Sakelliou \& Ponman (2006); (Do05) Donahue et al. (2005); (Mo05) Morris \& Fabian (2005); (Si09) Sivanandam et al. (2009); (Ke03) Kempner et al. (2003); (Cr08) Croston et al. (2008); (Le08) Leccardi \& Molendi (2008b); (Pr07) Pratt et al. (2007); (Wi04) Wise et al. (2004); (Vi05) Vikhlinin et al. (2005); (B107) Baldi et al. (2007); (Go04) Govoni et al. (2004); (Pe98) Peres et al. (1998); (An04) Andersson \& Madejski (2004); (Gu05) Gutierrez \& Krawczynski (2005); (Sm05) Smith et al. (2005); (Da02) Dahle et al. (2002); (Me04) Mercurio (2004); (Du08) Durret \& Lima Neto (2008); (Co06) Covone et al. (2006); (Ca09) Cavagnolo et al. (2009).

Donahue et al. (2005) define A1650 as a radio-quiet cool core, speculating that the entropy has been augmented by a recent AGN-triggered heating event also responsible for halting the AGN feeding process and ensuing radio manifestations. We classify A1650 as intermediate.

\subsubsection{A1689}

\section{Core classification}

Analysis of Chandra observations by Cavagnolo et al. (2009) show evidence of a well defined core with a metal abundance excess and a relatively high central entropy of about $80 \mathrm{keV} \mathrm{cm}^{2}$. We classify A1689 as an intermediate system.

\section{Dynamical classification}

Optical studies find evidence of two velocity peaks, possibly from line-of-sight superposition (Girardi \& Mezzetti 2001). This was later confirmed by Łokas et al. (2006), who performed a detailed kinematic study of about 200 galaxies with measured redshifts. Andersson \& Madejski (2004) find circumstantial evidence of a merger. We deem the evidence insufficient for classifying A1689 as a merger and conservatively catalog it as NOM.

\subsubsection{A963}

\section{Core classification}

Analysis of Chandra observations by Cavagnolo et al. (2009) show evidence of a well defined core with a modest temperature decrement, a metal abundance excess, and a relatively high central entropy of about $60 \mathrm{keV} \mathrm{cm}^{2}$. We classify A963 as an intermediate system.

\section{Entropy vs. alternative classification schemes}

In this section we compare our entropy classification scheme with the core and dynamical classification schemes presented in the previous section.

\subsection{Entropy vs. cool core classification scheme}

In Fig. 3 we compare our entropy (Sect. 4) and cool core (Sect. 5) classification schemes. We do this by plotting the temperature versus the emission measure ratio as in Fig. 2, with the nuance that we use colors to differentiate objects belonging to different classes; namely, we use red for non-cool core (NCC) systems, green for intermediate (INT) systems and blue for cool cores (CC). Inspection of Fig. 3 shows that, as expected, there is some correlation between the two classifications. More specifically, we find that 1) cool cores have low values of $\sigma$ or, in other words, are characterized by a strong pseudo-entropy gradient; 2) intermediate objects have intermediate entropy gradients; 3 ) non-cool core, on average, have small entropy gradients. We employ Fig. 3 to divide our objects into 3 broad entropy classes: low-entropy core (LEC) systems, medium-entropy core (MEC) systems and high-entropy core (HEC) systems. Given the continuous distribution of objects the precise values of $\sigma$ adopted to separate LEC from MEC and MEC from HEC are of course somewhat arbitrary. One possible criterion is that all INT objects belong to the MEC class. By adopting such a criterion, we set the separation between LEC and MEC at $\sigma=0.45$ and the separation between MEC and HEC at $\sigma=0.64$. In Table 2 we report the pseudo-entropy ratio and the entropy class for all objects in our sample. To help our readers navigate through the three different classification schemes, we provide in Table 4 a brief summary including the acronyms that are used extensively in this paper. 


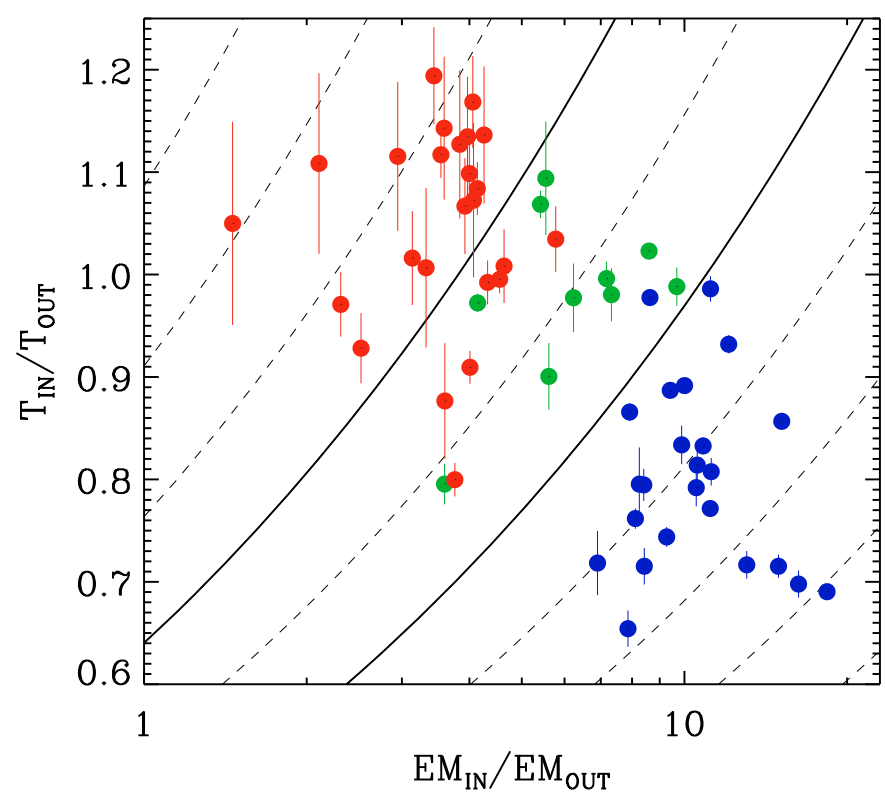

Fig. 3. Comparison of temperature and emission measure ratios for all clusters. The dashed and the solid curves indicate the regions of the plot where the pseudo-entropy ratio, $\sigma$, is constant; clusters with the strongest pseudo-entropy variations populate the bottom-right corner. The solid curves indicate the thresholds used to divide clusters in high (HEC), medium (MEC) and low (LEC) entropy core systems. Colors are associated to the cool core classification scheme: non-cool cores (NCC) are red, intermediate (INT) are green and cool cores (CC) are blue.

Table 4. Summary of classification schemes.

\begin{tabular}{lll}
\hline \hline Scheme & Classification & Acronym \\
\hline \multirow{3}{*}{ Entropy ratio } & Low-Entropy Core & LEC \\
& Medium-Entropy Core & MEC \\
& High-Entropy Core & HEC \\
& Cool Core & CC \\
Core properties & Intermediate & INT \\
& Non-Cool Core & NCC \\
\multirow{5}{*}{ Dynamic properties } & No Observed Merging & NOM \\
& Merging System & MRG \\
\hline
\end{tabular}

Interestingly, while the CC and INT systems separate out quite well in terms of their entropy ratios, the intermediate and non-cool core systems appear to be more mixed up. We find that only 1 CC systems is classified as an MEC and that 7 NCC systems are classified as MEC. The excellent match between LEC systems and cool cores is by no means a surprise: indeed, one of the possible definitions of a cool core cluster is that of a system hosting a low-entropy core (e.g. Cavagnolo et al. 2009). The agreement should instead be viewed as yet another demonstration of the effectiveness of the $\sigma$ indicator in describing the entropy profiles of clusters. To a lesser extent the same argument may be applied to the MEC vs. INT systems comparison and to the HEC and NCC comparison; however, for these systems, particularly for the latter, it becomes progressively more difficult to define the core and its properties. Indeed, the more attentive amongst our readers may recall that in Sect. 2 we refrained from including a number of clusters with poorly defined cores in our sample for the very reason that they would be difficult to classify.

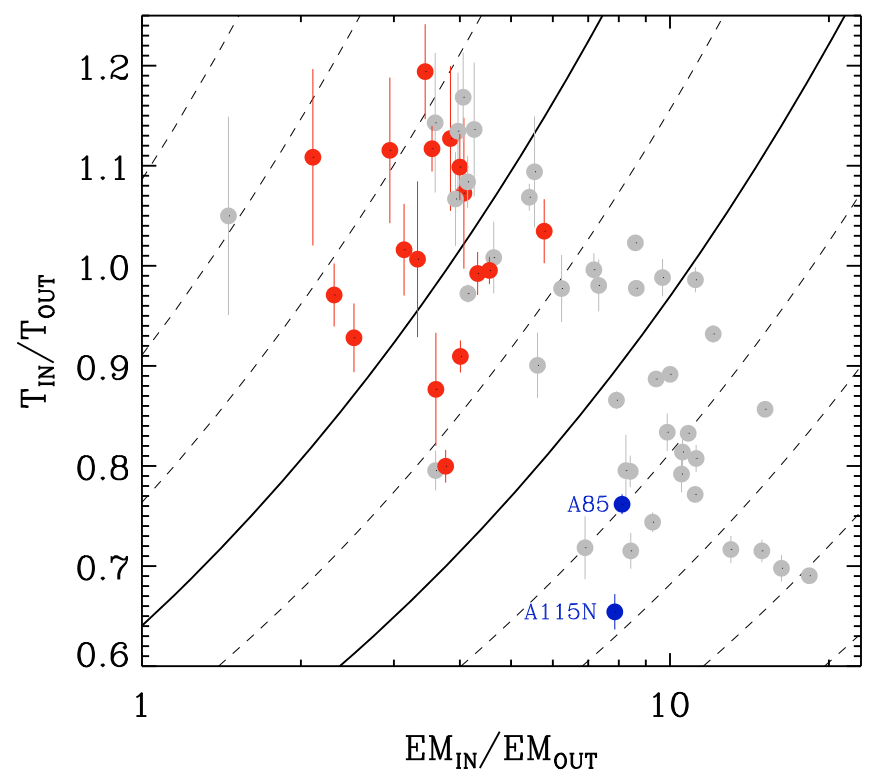

Fig. 4. Comparison of temperature and emission measure ratios for all clusters. The dashed and the solid curves indicate the regions of the plot where the pseudo-entropy ratio, $\sigma$, is constant; clusters with the strongest pseudo-entropy variations populate the bottom right hand corner. The solid curves indicate the thresholds used to divide clusters in high (HEC), medium (MEC) and low (LEC) entropy core systems. Colors are associated to the dynamics-based classification scheme, namely mergers (MRG) are red, with the exception of A115N and A85, which are blue, and systems that do not show evidence of merging (NOM) are gray.

\subsection{Entropy vs. dynamical classification scheme}

In Fig. 4 we compare our entropy (Sect. 4) and dynamical (Sect. 5) classification schemes. We do this as in Fig. 3 with the difference that the color coding now refers to the dynamical classification, more specifically red for merging (MRG) systems, with the exception of $\mathrm{A} 115 \mathrm{~N}$ and $\mathrm{A} 85$, which are blue, and gray for systems that do not show evidence of merging (NOM). Inspection of Fig. 4 shows that there is some correlation between the two classifications. More specifically we find that: 1) the majority of mergers are HEC systems, a few are classified as MEC systems, and 2 are LEC systems; 2) NOM systems are found all over the plot. The result for NOM systems has a trivial explanation, as already discussed in Sect. 5, these are objects for which we do not observe evidence of merging: they will include both systems that are not undergoing a merger and mergers for which we do not have observational evidence of merging activity. A more interesting result is the one on objects identified as mergers. As already noted, only a few mergers are MEC and LEC, so we now focus on the 2 MRG with the lowest pseudoentropy ratios. These are A85 and A115N, which are both LEC systems. A85 and A115N, which are plotted in blue in Fig. 4, are systems where X-ray observations clearly show two clumps. In both cases the evidence found in the literature supports a scenario where the effects of the merger have not reached the core of the main structure (the one for which we computed the entropy ratio), either because the merger is an off-axis merger, A115N (Gutierrez \& Krawczynski 2005), or because it is in an early stage, A85 (Kempner et al. 2002) and A115N (Barrena et al. 2007b). A potential concern for these objects is that, if the sole evidence that the merger has not reached the core were the presence of the core itself, the whole argument would of course be circular and not particularly convincing. We note that if the 
mergers were in an advanced state, we would expect distorted morphology and irregular temperature distribution in the circumcore regions, as well as substantial displacement between X-ray and optical light peaks. This is indeed what is observed in other merging systems (e.g. A2256, Sun et al. 2002; and Bourdin \& Mazzotta 2008; A3667, Briel et al. 2004; and Vikhlinin et al. 2001) and predicted in simulations (e.g. Rowley et al. 2004; Ricker \& Sarazin 2001). In the cases of A85, no such evidence is found. In the case of A115N, Gutierrez \& Krawczynski (2005) find evidence for heating of the region separating the cores, but no indication of supersonic motion; moreover, Barrena et al. (2007b) detect two optical substructures of cluster-type that are well recognizable in the plane of the sky and roughly coincident with the X-ray peaks, thereby favoring a pre-merger scenario. Therefore, in A115N, we have evidence of some form of interaction of minor intensity, which may be explained either in the context of an off-axis merger or in that of an early stage of the merger process.

It is quite interesting that, when comparing our core entropy based classification with the dynamical classification, the only 2 mergers to possess an LEC are systems where the effects of the merger have not reached the core. The rather obvious inference, which is discussed at some length later in the paper (see Sect. 7.2), is that mergers do have the capability of disrupting low-entropy cores. For the time being we note that, if we exclude those interacting systems for which the effects of the merger have not reached the core, we find that MRG systems have pseudo-entropy ratios greater than 0.51 . A potential concern is that the same observational evidence may have been used to classify an object as a merger and as an HEC, this would of course provide a rather trivial explanation for the correlation between the two classifications. We note that the presence of a well defined core does not imply that an object may not also show substructure in its surface brightness and temperature maps, indeed A1644 (Reiprich et al. 2004) and A115N (Gutierrez \& Krawczynski 2005) are both good examples of such systems. Moreover, only 3 out of the 17 bona-fide mergers(we excluded the 2 special cases of $\mathrm{A} 85$ and A115N) have been classified as mergers on the basis of their X-ray properties alone; for the other 14 systems, there is evidence of a merger from radio and/or optical observations.

In summary the comparison of our entropy and dynamics based classification schemes shows that dynamically active systems tend to have high-entropy cores while low-entropy cores are not found in merging systems, with the exception of A85 and $\mathrm{A} 115 \mathrm{~N}$, where the effects of the mergers have not reached the core.

\subsection{Comparison with previous work}

Ours is not the first attempt to divide clusters on the basis of their core properties. There have been various works concentrating on somewhat different core properties. Sanderson et al. (2006), for instance, consider the core temperature as discriminator and define as cool core clusters those systems for which the ratio between average cluster and core temperature exceeds unity at greater than $3 \sigma$ significance. The average cluster temperature is determined from an annulus with bounding radii $0.1-0.2 R_{500}$ and the core temperature from a circle with radius $0.1 R_{500}$. The circle is similar to our inner region, while the annulus is somewhat smaller than our outer region. While the selection procedure appears to work well for the specific objects in the Sanderson et al. (2006) sample, it has some rather obvious pitfalls. Visual inspection of Fig. 3 shows that the range

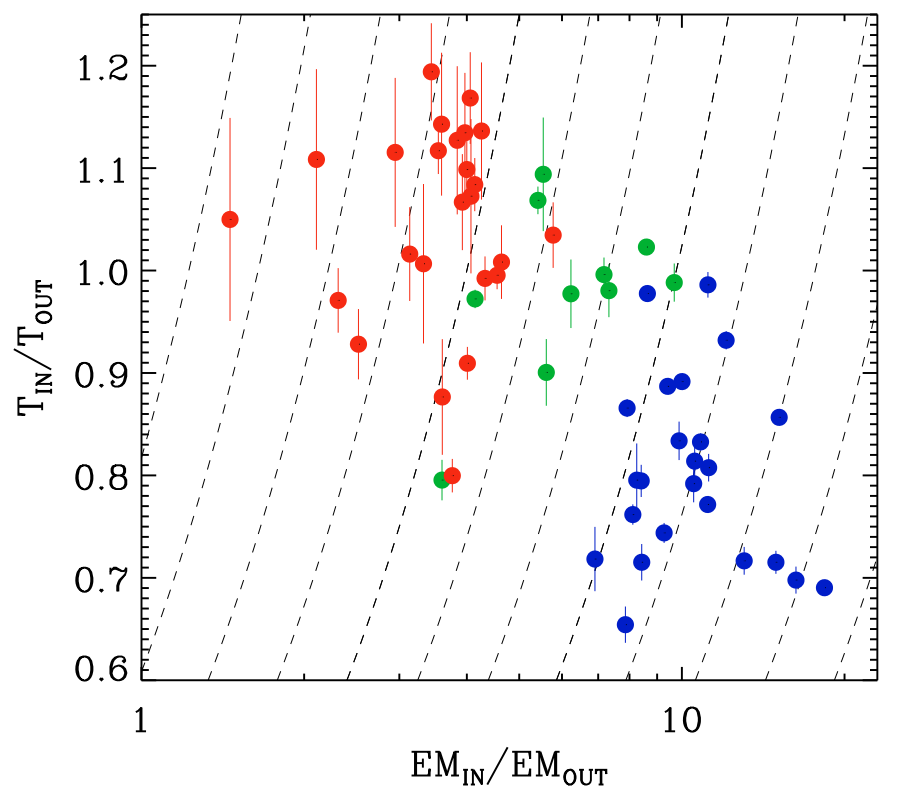

Fig. 5. Comparison of temperature and emission measure ratios for all clusters. The dashed curves indicate the regions of the plot where the pseudo-cooling-time ratio, $\tau$, defined in Eq. (2), is constant. Clusters with the strongest pseudo- cooling-time variations populate the bottomright corner. Colors are associated to the cool core classification scheme, namely the non-cool cores (NCC) are red, intermediate (INT) are green, and cool cores (CC) are blue.

$0.8<T_{\text {IN }} / T_{\text {OUT }}<1.0$ is populated by objects belonging to all 3 entropy classes, i.e. LEC, MEC, and HEC, it is only the additional use of the $E M_{\mathrm{IN}} / E M_{\mathrm{OUT}}$ ratio that allows us to provide a more effective means of separation. As an example of the limitations associated to a classification system based on the temperature decrement alone, we may consider Fig. 4 where we observe that, in contrast to what is found when employing the entropy classification scheme, a sizeable fraction of mergers are found in clusters without temperture decrement.

Baldi et al. (2007) use the cooling time, or better the ratio of cooling time to age of the universe at the cluster redshift. As already noted in Sect. 4, a pseudo-cooling-time ratio, $\tau$ defined as in Eq. (2), separates out clusters in much the same way as the pseudo-entropy-ratio does. This is illustrated in Fig. 5 where we show the same plot reported in Fig. 3 with the only difference that the dashed lines indicate region of constant $\tau$ rather than $\sigma$.

Other authors have compared a core-based classification with a dynamics-based classification. McCarthy et al. (2004) divide objects in cooling flow and non-cooling flow depending on the presence or absence of a temperature gradient in their cores. This is essentially the same classification adopted by Sanderson et al. (2006). McCarthy et al. (2004) also provide a dynamical classification dividing their objects in relaxed and non-relaxed on the basis of the presence or absence of large-scale (a few hundred $\mathrm{kpc}$ ) substructure in the X-ray images, presumably related to mergers. They find that, of the 33 objects in their sample, 18 are cooling-flow and 15 non-cooling flow. Interestingly, of the 18 cooling flow objects, 12 are classified as relaxed and 6 as nonrelaxed, and of the 15 non-cooling flow objects 9 are non-relaxed and 6 are relaxed. The presence of a sizable number of nonrelaxed cooling flow systems and of relaxed non-cooling flow systems is considered as evidence for a different origin of cooling flows and the relaxed/non-relaxed state in clusters. McCarthy et al. (2004) propose a scenario where an object ends up being cooling or non-cooling flow on the basis of the amount of 
entropy injected in the system, while the relaxed or non-relaxed nature depends on the object having or not having recently experienced a merger. Our own findings appear to be at variance with what is reported by McCarthy et al. (2004): the objects that we classify as mergers, with the exception of systems where the effects of the merger have not reached the core, are all characterized by a high pseudo-entropy ratio.

Since 15 of the 33 object in McCarthy's sample are also found in ours, we compared our results with theirs ${ }^{5}$. For 11 of the 15 objects that are in common, their classification agrees with ours, more specifically: 8 objects are classified as cooling flow and relaxed by McCarthy et al. (2004) and as LEC and NOM by ourselves; 2 objects are classified as non-cooling flow and non relaxed by McCarthy et al. (2004) and as nonlow-entropy systems, either HEC and MEC, and mergers by ourselves; one object, namely A115N, is classified as cooling flow and non-relaxed by McCarthy et al. (2004) and as LEC and MRG by ourselves. For 4 objects their classification appears to differ from ours. More specifically, there is one object, namely A1068, which we classify as LEC and non-merging and which McCarthy et al. (2004) classify as cooling flow and non-relaxed. McCarthy et al. (2004) refer to a paper by Wise et al. (2004), which indeed provides evidence for substructure in surface brightness, temperature, and metal abundance; however, all images presented in that paper cover a region of $200 \mathrm{kpc} \times 200 \mathrm{kpc}$ and therefore only include the cool core. The kind of substructure found in the core of A1068 is akin to that found in many other cool core systems and is generally believed to be associated to the AGN found at the center of these systems and not to a merger. A second object, A85, is classified as LEC and MRG by ourselves and as non-cooling flow and non-relaxed by McCarthy et al. (2004); however, the authors do not specify whether the core property is referred to the main structure, which is a well known cool core (e.g. Peres et al. 1998) or to the substructure that hosts an intermediate core (Kempner et al. 2002); assuming they refer to the latter, the difference in core classification is trivial as we refer to the main structure.

The last 2 objects, A1413 and A1689, are classified by McCarthy et al. (2004) as non-cooling flow and relaxed, while we classify them as MEC and NOM. A first important point is that McCarthy et al. (2004) define as relaxed those objects for which they do not find evidence of large-scale irregularities in the X-ray images. This however, does not necessarily imply that these objects are indeed relaxed (an example of such relatively rare systems is A401, a cluster with fairly regular X-ray morphology, Sakelliou \& Ponman 2004, featuring a small radio halo, Bacchi et al. 2003, and significant structure in X-ray temperature, Sakelliou \& Ponman 2004; and Bourdin \& Mazzotta 2008). We classify A1413 and A1689 as MEC in our entropy classification system; both these systems possess a well defined core that, however, is not as prominent as the ones typically found in LEC systems. Indeed analysis of Chandra observations of A1413 and A1689 provide evidence of a well defined core with a modest temperature drop in the core (Vikhlinin et al. 2005; Cavagnolo et al. 2009), a metal abundance excess (Vikhlinin et al. 2005; Cavagnolo et al. 2009), and a relatively high central entropy of about $60 \mathrm{keV} \mathrm{cm}^{2}$ and $80 \mathrm{keV} \mathrm{cm}^{2}$, respectively (Cavagnolo et al. 2009).

In summary, by comparing our classification schemes with those provided by McCarthy et al. (2004), we find that, of the

\footnotetext{
5 In this comparison we only make use of our dynamical and entropy classifications, the cool core classification is omitted as it provides identical results to those derived from the entropy classification.
}

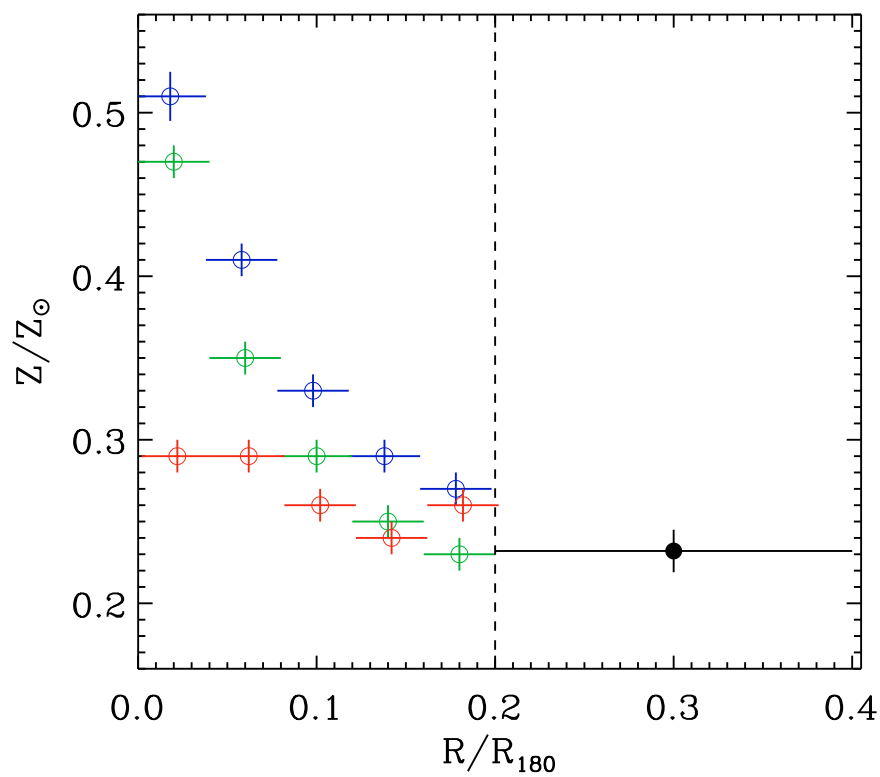

Fig. 6. Mean metallicity profiles for LEC (blue circles), MEC (green), and HEC (red) clusters. Abundances are expressed in Anders \& Grevesse (1989) solar values. The dashed line indicates $0.2 R_{180}$, the radius within which profiles are obtained from all clusters and the black data point beyond $0.2 R_{180}$ is the average value from distant (i.e. $z>0.1$ ) clusters belonging to all entropy classes.

4 objects for which we do not agree, the 3 objects with mixed classifications i.e. mergers with cooling flows (A1068) and relaxed non-cooling flows (A1413 and A1689) cannot be used to support a scenario where the absence or presence of a cooling flow is unrelated to the object having or not having experienced a merger. Indeed, the former object is non-relaxed on small scales in much the same way as other cool core systems and for the last two: I) the lack of evidence for a merger does not necessarily mean that a merger is not present; II) the classification as noncooling flow is insufficiently accurate, as both these system host well defined intermediate cores.

\section{Chemical properties}

In this section we compare chemical with thermo-dynamic properties for the objects in our sample. As a first step we discuss the metal abundance profiles.

\subsection{Metallicity profiles}

We have divided our sample in the entropy classes defined in Sect. 6 and produced mean radial metallicity profiles for each entropy class. Metal abundance profiles for individual systems come from Leccardi \& Molendi (2008a) for the intermediate redshift subsample and from Rossetti \& Molendi (2010) from the low redshift sample. In Fig. 6 we show the mean profiles for LEC, MEC, and HEC clusters. The binning is in units of $R_{180}$ and was computed as in Leccardi \& Molendi (2008a), and for each bin, the average is calculated by allowing for an intrinsic dispersion. As can be seen in Fig. 6, within $0.1 R_{180}$, a region typically associated with the core, all profiles show an abundance excess. The excess is strongest for LEC, somewhat weaker for MEC, and weakest for HEC clusters. Interestingly, the modest excess observed in HEC clusters is similar to the one obtained from a BeppoSAX sample of non-cool core clusters (De Grandi \& Molendi 2001) dominated by well known merging systems. 
Between 0.1 and $0.2 R_{180}$, the profiles for the three classes are roughly consistent with one another and, at least for LEC and MEC systems, show a significant excess with respect to the mean value measured in the outskirts. Between 0.2 and $0.4 R_{180}$, where we only have data for the intermediate-redshift sample, the three profiles are consistent with being flat and equal with each other. We therefore plot the abundance averaged over all 3 entropy classes, which turns out to be $0.23 \pm 0.01 Z_{\odot}$, in good agreement with the value obtained by De Grandi \& Molendi (2001) for a local sample of relaxed clusters observed with BeppoSAX. As already discussed in Sect. 4, the precise choice of pseudo entropy ratio values used to divide objects in the three entropy classes is rather arbitrary. By experimenting with slightly different values we find that, while the details of the profiles may change somewhat as borderline objects are shifted from one class to another, the qualitative description provided above remains valid.

An important point is that the abundance measures in the outer region are not only consistent with being flat but also appear to be independent of the entropy class (HEC, MEC, or LEC) or of the dynamical class (MRG or NOM) of the object. Moreover the mass of ICM enclosed within 0.2 and $0.4 R_{180}$ is about two times that contained within $0.2 R_{180}$ and, according to De Grandi et al. (2004), the Fe mass in the abundance excess of CC clusters is roughly $10 \%$ of the Fe mass integrated out to $0.25 R_{180}$. It follows that estimates of how the global metal abundance varies with respect to other quantities are best performed by making measures in the $0.2-0.4 R_{180}$ range. An example, which we shall not discuss further, is the often quoted anti-correlation between metal abundance and temperature (Baumgartner et al. 2005; Balestra et al. 2007). Another example is the measure of the evolution of the global metal abundance with cosmic time. Current estimates (Balestra et al. 2007; Maughan et al. 2008) are performed at small radii where the abundance excess, more pronounced in some systems than in others, poses a major obstacle. Both Balestra et al. (2007) and Maughan et al. (2008) are aware of these difficulties and confront them either by gauging how the mix of cool cores and non-cool cores might affect the observed evolution in the iron abundance (Balestra et al. 2007), or by excising the innermost region $\left(0.15 R_{500}\right)$ from their spectra (Maughan et al. 2008). A more robust approach would be to restrict measures to the $0.2-$ $0.4 R_{180}$ range. In Leccardi \& Molendi (2008a), we showed that by adopting the above radial range in the limited redshift interval covered by our data, $0.1<z<0.3$, we could not distinguish between no variation in the abundance with redshift and a variation of the kind described in Balestra et al. (2007). Extension of this kind of measure out to $z \simeq 0.5$, while observationally challenging, would allow to distinguish between the two competing alternatives.

\subsection{Chemical vs. thermo-dynamic quantities}

In Fig. 7 we plot the metallicity measured in our inner region, $Z_{\mathrm{IN}}$, vs. the pseudo-entropy ratio, $\sigma$, for all clusters in our sample. Values of $Z_{\mathrm{IN}}$ and $\sigma$ are also reported in Table 2. We find $\sigma$ and $Z_{\mathrm{IN}}$ to have a negative correlation: the stronger the pseudoentropy gradient, the stronger the metallicity peak. This results tells us that, barring a few exceptions that we discuss later in this section, the metal rich gas in clusters also happens to be the lowentropy gas. An important point that we only mention here and that we have discussed in detail elsewhere is the presence of a consistent scatter in the abundance distribution, particularly for LEC systems (De Grandi \& Molendi 2009). The most interesting result is arguably the presence of a few (7) MEC and HEC

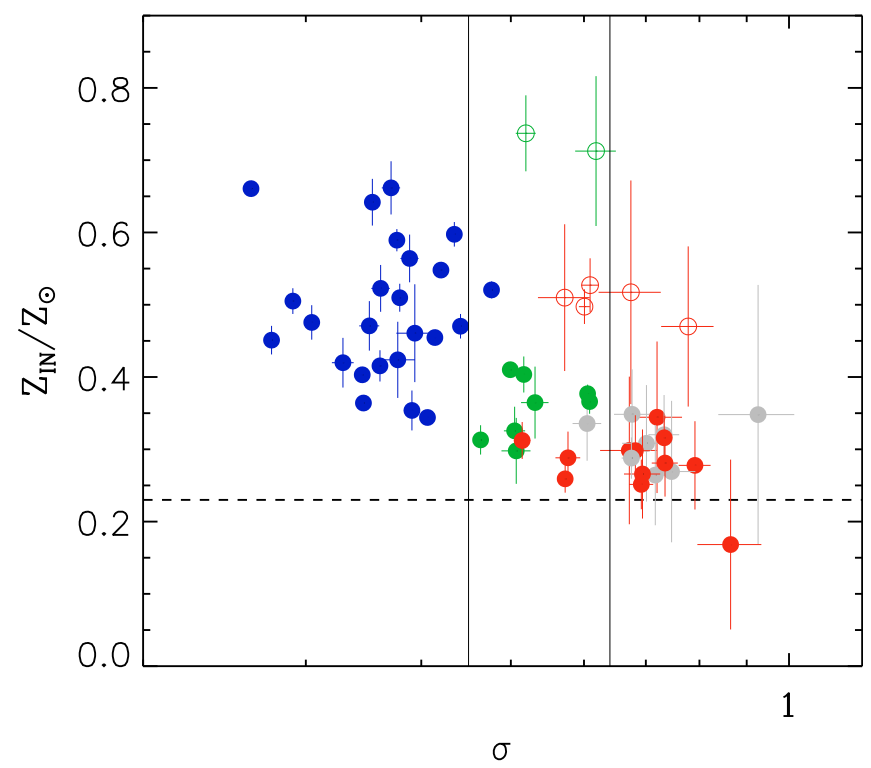

Fig. 7. Central metallicity, $Z_{\mathrm{IN}}$, vs. pseudo-entropy ratio, $\sigma$. In blue and green we indicate objects that, within the core classification scheme, are respectively identified as CC and INT systems. In red and gray we indicate objects that, within the dynamical classification scheme, are respectively identified as MRG and NOM. In the few instances where the two classification schemes require different colors, the core scheme takes precedence. Open circles indicate high metallicity MEC and HEC systems. Abundances are expressed in Anders \& Grevesse (1989) solar values. The dashed horizontal line at $0.23 Z_{\odot}$ indicates the mean metallicity in the outer regions of clusters (Leccardi \& Molendi 2008a). The solid vertical lines mark the boundary between the three entropy classes (see Sect. 6.1 for details).

systems with unusually high metal abundance. Of these high $Z$ systems 4, namely A1644, AS0084, A576, and A3562, have metalicities well above the typical values found for other MEC and HEC systems, while 3, namely A2034, A209, and A1763, suffering from large indetermination in there abundance estimates, show poor statistical evidence of an excess (roughly $2 \sigma$ ). For A2034, a long Chandra observation (Baldi et al. 2007) provides a somewhat tighter constraint that raises the significance to about $3 \sigma$.

From our classification work (see Sect. 5), we know that a large fraction of these objects, 5/7, are mergers (shown in red in Fig. 7). In other words, most of our high metallicity MEC and HEC systems are undergoing a phase of rapid dynamical change. This simple consideration leads to the question of the original equilibrium configuration from which these systems evolved. There are two issues that should be kept in mind when addressing this question. The first is that metals are reliable markers of the ICM, in the sense that, once metals have polluted a given region of a cluster, the timescale over which the same metals will diffuse is comparable, likely longer, than the Hubble time (Sarazin 1988; Chuzhoy \& Nusser 2003). Thus, for all practical purposes, metals trace the region of the ICM where they have been injected and can be diluted only if the ICM itself undergoes mixing processes. The second is that abundances, such as those observed in our metal rich MEC and HEC systems, are found in the cores of LEC systems, indeed our own analysis (see Fig. 7) shows that every LEC system has an excess with respect to the metal abundance found in cluster outer regions.

Keeping the above considerations in mind, the most likely explanation is that our high abundance MEC and HEC clusters originate from LEC systems that have undergone substantial 
heating. While for one of our objects, namely A1644, the relatively modest entropy ratio is not inconsistent with heating from the central AGN, as observed in other intermediate systems such as A1650 (Donahue et al. 2005). For all other systems, the required heating is beyond what can be provided by the central AGN and must come from some other mechanism. Since all but one of the other 6 high metallicity objects show evidence of a merger, it seems reasonable to assume that the heating may indeed be provided by the merger event.

This interpretation, however, clashes with claims from at least 2 groups conducting cluster simulations. According to Poole et al. (2006, 2008) and Burns et al. (2008), once cool cores form it is extremely difficult to disrupt them. The above authors suggest that the fate of a cluster (LEC or otherwise) is decided early on in its history; if it is subject to an event that raises its entropy then it will likely not develop a cool core and subsequent mergers will be effective in maintaining the high-entropy state. Conversely, if a cool core is formed early on, it will be very difficult to disrupt; i.e., subsequent mergers will not destroy the entropy stratification. In our opinion, the scenario described in Poole et al. (2006, 2008) and Burns et al. (2008) suffers from two major shortcomings, one on the observational side, the other on the theoretical side. Let us consider the former: if the presence/absence of cool cores is not related to the dynamical state of a cluster, one would expect to observe LEC in some merging clusters, and HEC in some relaxed systems. In Sect. 5 we have shown that 1) having excluded A85 and A $115 \mathrm{~N}$, where the effects of the merger have not reached the core, for none of the remaining 21 LEC clusters do we find evidence that they are located in a merging system; 2) all our merging systems, barring the afore quoted exceptions of A85 and A115N, are MEC or HEC systems, with the vast majority being HEC (11/17). These findings are at variance with those reported by McCarthy et al. (2004), who do identify a few non-relaxed cooling-flow systems and relaxed non-cooling-flow systems. In Sect. 6.3 we compared our entropy and dynamical classification schemes with those presented in McCarthy et al. (2004) for the 15 objects that are present in both samples finding that, of the 4 cases where our classifications do not agree, 3 likely result from misclassifications by McCarthy et al. (2004), while the fourth probably has a trivial explanation.

The second shortcoming is related to the current simulations not being able to reproduce observed cool cores, conversely they produce something more akin to traditional cooling flows. Poole et al. $(2006,2008)$ correct for this by imposing initial conditions in the core so as to reproduce observed cool cores; however, merger events run on timescales longer than the cooling time in cores. Indeed, as pointed out in Poole et al. (2006), within $0.5 \mathrm{Gyr}$, well before the clusters begin interacting significantly, the central entropy profile reverts to the self-similar power-law shape. Alternatively, Burns et al. (2008) strive to reproduce observed cool cores by introducing ad-hoc sub-grid recipes. Thus, if simulations cannot provide a self-consistent picture of cool cores, why should we be compelled to trust simulations that tell us that cool cores survive mergers?

Recently Sanderson et al. (2009) have provided observational evidence favoring scenarios where cluster mergers are capable of erasing cool cores. These authors have shown that, in a sample of 65 objects, the X-ray/BCG projected offset correlates with the gas density profile. Under the assumption that the offset serves to measure the dynamical state of the cluster, their result implies that the cool core strength progressively diminishes in more dynamically disrupted clusters. Such a trend is expected if cluster mergers are capable of erasing cool cores.

\section{Summary}

The main results presented in this paper may be summarized as follows.

- We have constructed an indicator of the entropy of the core relative to that of the cluster, the pseudo entropy ratio $\sigma$. Our indicator is robust, in the sense that somewhat different choices of the quantities from which the ratio is computed result in very similar values of $\sigma$. The indicator is also relatively parsimonious, in the sense that it may be constructed from data of moderate statistical quality.

- The classification of clusters based on the entropy indicator improves upon the traditional classification scheme based on the presence or absence of a temperature drop in the core. Conversely, classification schemes based on the central cooling time appear to be essentially equivalent to ours.

- A comparison between the entropy-based classification scheme and a classification scheme based on dynamical properties shows that the large majority of merging systems are characterized by large entropy ratios. Only 2 of our merging systems feature a low-entropy core (LEC), and in both cases we were able to establish, with reasonable certainty, that the effects of the merger have not reached the core. Our findings are at variance with those presented by McCarthy et al. (2004), who do find evidence of non relaxed cooling flow systems and relaxed non-cooling flow systems. We have compared our entropy and dynamical classification schemes with those in McCarthy et al. (2004) for the 15 objects that are common to both samples and find that the 3 cases where our classifications do not agree probably result from misclassifications by McCarthy et al. (2004).

- We find that mean abundance profiles for our 3 entropy classes, namely low-entropy core (LEC), medium-entropy core MEC, and high-entropy core (HEC), may be divided in 3 regions. In the outer region, between 0.2 and $0.4 R_{180}$, all 3 profiles are consistent with a flat line and with one another. In the core region, within $0.1 R_{180}$, all classes feature an excess with respect to the mean value found in the outer regions. The excess is strongest for LEC, somewhat weaker for MEC, and weakest for HEC clusters. Between 0.1 and $0.2 R_{180}$, the profiles for the three classes are roughly consistent with one another and, at least for LEC and MEC systems, show a significant excess with respect to the mean value measured in the outskirts.

- We find that objects with stronger pseudo-entropy gradients have more pronounced metallicity peaks. This result tells us that, barring a few exceptions, the gas that is more enriched in metals also happens to be the one featuring the lowest entropy.

- We have identified a small number of medium and high entropy core systems with high central metallicity. The majority of these objects have been classified as mergers, i.e. as systems far from their equilibrium configuration. We surmise that these systems evolved from low-entropy core clusters that have experienced a heating event. We examined simulation-based claims that conflict with our conjecture, finding they are flawed both on the observational and the theoretical sides.

In an associated paper (Rossetti \& Molendi 2010) we will further investigate the issue of medium and high-entropy core systems with a large central abundance. We will do this by performing bi-dimensional analysis of a smaller sample of bright and nearby clusters. 

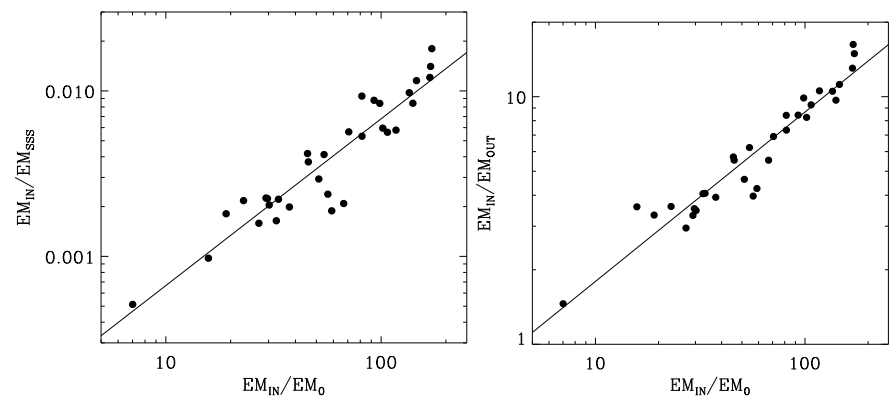

Fig. A.1. Correlations between various definitions for the $E M$ ratios (see text for details). Left panel: self-similar scaled vs. ideal ratios. Right panel: standard vs. ideal ratios. The solid curve is the best fit with a power law. The uncertainties are smaller than the point size. The scatter is $29 \%$ and $16 \%$ for left and right panels, respectively.

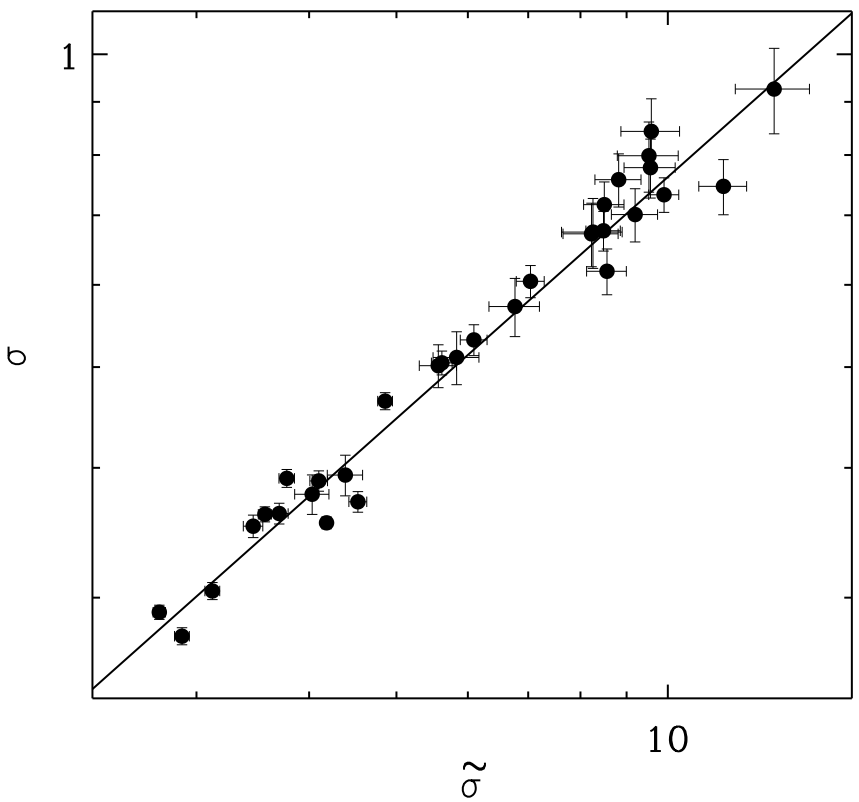

Fig. A.2. Correlation between $\sigma$ and $\tilde{\sigma}$ (see text for definitions) for clusters with $z>0.1$. The solid line shows the best-fit power law; the scatter is $\approx 6 \%$.

Acknowledgements. We acknowledge financial support from contracts ASI-INAF $\mathrm{I} / 023 / 05 / 0$ and $\mathrm{I} / 088 / 06 / 0$. This research has made use of two databases: the NASA/IPAC Extragalactic Database (NED) and the X-Ray Cluster Database (BAX) and of three archives: the High Energy Astrophysics Science Archive Research Center (HEASARC), the XMM-Newton Science Archive (XSA), and the Archive of Chandra Cluster Entropy Profile Tables (ACCEPT). We would like to express our appreciation for the excellent work by Cavagnolo and collaborators in setting up the ACCEPT archive. We acknowledge useful discussions with Stefano Ettori, Stefano Borgani. We thank Sabrina De Grandi and Fabio Gastaldello for a careful and critical reading of the manuscript.

\section{Appendix A:}

In this Appendix we compare $E M_{\mathrm{OUT}}$, the emission measure from the $0.05-0.20 R_{180}$ ring, with another proxy for the emission measure at large radii, namely the self-similar scaling factor $E M_{\text {SSS }}$, which is defined as

$$
E M_{\mathrm{SSS}} \equiv \Delta_{\mathrm{Z}}^{3 / 2}(1+z)^{9 / 2}\left(k T_{\mathrm{OUT}} / 10 \mathrm{keV}\right)^{1 / 2}
$$

For details about this definition we refer our readers to Arnaud et al. (2002). As a reference value of the emission measure at large radii, we employ $E M_{0}$, the emission measure calculated in the $0.2-0.4 R_{180}$ ring, $E M_{0}$ is available for the subsample of distant $(z>0.1)$ clusters, for which it is possible to measure $E M$ out to $0.4 R_{180}$ (values of $E M_{0}$ are from Leccardi \& Molendi $2008 b$ ). Since we are interested in emission measure ratios, for each distant cluster, we calculated our "ideal" ratio $E M_{\mathrm{IN}} / E M_{0}$, determined directly from the data, the self-similar scaled ratio $E M_{\mathrm{IN}} / E M_{\mathrm{SSS}}$, and the standard ratio $E M_{\mathrm{IN}} / E M_{\mathrm{OUT}}$. In Fig. A.1 we compare the self-similar scaled (left panel) and the standard (right panel) ratios to the ideal ratio. We find a good correlation for both cases, but the scatter is smaller (16\% vs. 29\%) when using the standard ratio. Throughout this paper we make use of $E M_{\mathrm{IN}} / E M_{\mathrm{OUT}}$, but we emphasize that our results are largely independent of this particular choice. Indeed, the appearance of the plot in Fig. 2 is very similar when using

$$
\tilde{\sigma} \equiv\left(T_{\mathrm{IN}} / T_{\mathrm{OUT}}\right) \times\left(E M_{\mathrm{IN}} / E M_{\mathrm{SSS}}\right)^{-1 / 3},
$$

which differs from $\sigma$ for the use of the self-similar scaling. In Fig. A. 2 we show the correlation between $\sigma$ and $\tilde{\sigma}$ for the subsample of distant clusters. We find a small, $\approx 6 \%$, scatter around the best-fit power law (represented with a solid line), this is another confirmation of the robustness of our entropy indicator.

\section{References}

Abell, G. O. 1958, ApJS, 3, 211

Abell, G. O. 1965, ARA\&A, 3, 1

Abell, G. O. 1975, Clusters of Galaxies, ed. A. Sandage, M. Sandage, \&

J. Kristian (the University of Chicago Press), 601

Anders, E., \& Grevesse, N. 1989, Geochim. Cosmochim. Acta, 53, 197

Andersson, K. E., \& Madejski, G. M. 2004, ApJ, 607, 190

Arnaud, M., Aghanim, N., \& Neumann, D. M. 2002, A\&A, 389, 1

Bacchi, M., Feretti, L., Giovannini, G., \& Govoni, F. 2003, A\&A, 400, 465

Bagchi, J., Durret, F., Neto, G. B. L., \& Paul, S. 2006, Science, 314, 791

Baldi, A., Ettori, S., Mazzotta, P., Tozzi, P., \& Borgani, S. 2007, ApJ, 666, 835

Balestra, I., Tozzi, P., Ettori, S., et al. 2007, A\&A, 462, 429

Bardelli, S., Pisani, A., Ramella, M., Zucca, E., \& Zamorani, G. 1998, MNRAS, 300, 589

Barrena, R., Boschin, W., Girardi, M., \& Spolaor, M. 2007a, A\&A, 467, 37

Barrena, R., Boschin, W., Girardi, M., \& Spolaor, M. 2007b, A\&A, 469, 861

Bauer, F. E., Fabian, A. C., Sanders, J. S., Allen, S. W., \& Johnstone, R. M. 2005, MNRAS, 359, 1481

Baumgartner, W. H., Loewenstein, M., Horner, D. J., \& Mushotzky, R. F. 2005, ApJ, 620, 680

Blanton, E. L., Sarazin, C. L., \& McNamara, B. R. 2003, ApJ, 585, 227

Bourdin, H., \& Mazzotta, P. 2008, A\&A, 479, 307

Bravo-Alfaro, H., Caretta, C. A., Lobo, C., Durret, F., \& Scott, T. 2009, A\&A, 495, 379

Briel, U. G., Finoguenov, A., \& Henry, J. P. 2004, A\&A, 426, 1

Buote, D. A., \& Tsai, J. C. 1995, ApJ, 452, 522

Buote, D. A., \& Tsai, J. C. 1996, ApJ, 458, 27

Burgett, W. S., Vick, M. M., Davis, D. S., et al. 2004, MNRAS, 352, 605

Burns, J. O., Hallman, E. J., Gantner, B., Motl, P. M., \& Norman, M. L. 2008, ApJ, 675, 1125

Cassano, R., Brunetti, G., \& Setti, G. 2006, MNRAS, 369, 1577

Cassano, R., Brunetti, G., Venturi, T., et al. 2008, A\&A, 480, 687

Cavagnolo, K. W., Donahue, M., Voit, G. M., \& Sun, M. 2009, ApJS, 182, 12

Chen, Y., Reiprich, T. H., Böhringer, H., Ikebe, Y., \& Zhang, Y.-Y. 2007, A\&A, 466,805

Choi, Y.-Y., Reynolds, C. S., Heinz, S., et al. 2004, ApJ, 606, 185

Chuzhoy, L., \& Nusser, A. 2003, MNRAS, 342, L5

Clarke, T. E., Blanton, E. L., \& Sarazin, C. L. 2004, ApJ, 616, 178

Covone, G., Adami, C., Durret, F., et al. 2006, A\&A, 460, 381

Croston, J. H., Pratt, G. W., Böhringer, H., et al. 2008, A\&A, 487, 431

Dahle, H., Kaiser, N., Irgens, R. J., Lilje, P. B., \& Maddox, S. J. 2002, ApJS, 139,313

David, L. P., \& Nulsen, P. E. J. 2008, ApJ, 689, 837

David, L. P., Nulsen, P. E. J., McNamara, B. R., et al. 2001, ApJ, 557, 546

De Grandi, S., \& Molendi, S. 2001, ApJ, 551, 153

De Grandi, S., \& Molendi, S. 2009, A\&A, 508, 565

De Grandi, S., Ettori, S., Longhetti, M., \& Molendi, S. 2004, A\&A, 419, 7

Dickey, J. M., \& Lockman, F. J. 1990, ARA\&A, 28, 215 
Dixon, W. V. D., Kriss, G. A., Ferguson, H. C., \& Malumuth, E. M. 1987, BAAS, 19,1080

Donahue, M., Voit, G. M., O’Dea, C. P., Baum, S. A., \& Sparks, W. B. 2005 ApJ, 630, L13

Dunn, R. J. H., Fabian, A. C., \& Taylor, G. B. 2005, MNRAS, 364, 1343

Dupke, R. A., Mirabal, N., Bregman, J. N., \& Evrard, A. E. 2007, ApJ, 668, 781

Durret, F., Lima Neto, G. B., \& Forman, W. 2005, A\&A, 432, 809

Durret, F., \& Lima Neto, G. B. 2008, Adv. Space Res., 42, 578

Edge, A. C., Stewart, G. C., Fabian, A. C., \& Arnaud, K. A. 1990, MNRAS, 245, 559

Ettori, S., Fabian, A. C., Allen, S. W., \& Johnstone, R. M. 2002, MNRAS, 331, 635

Fadda, D., Biviano, A., Marleau, F. R., Storrie-Lombardi, L. J., \& Durret, F. 2008, ApJ, 672, L9

Feretti, L., Fusco-Femiano, R., Giovannini, G., \& Govoni, F. 2001, A\&A, 373, 106

Feretti, L., Giovannini, G., \& Bohringer, H. 1997, New Astron., 2, 501

Finoguenov, A., Henriksen, M. J., Briel, U. G., de Plaa, J., \& Kaastra, J. S. 2004, ApJ, 611, 811

Feretti, L., \& Giovannini, G. 2007, in Panchromatic view of clusters of galaxies and the large-scale structure, Springer Lect. Notes in Phys.

Ghizzardi, S., Rossetti, M., \& Molendi, S. 2009, A\&A, submitted

Giacintucci, S., Venturi, T., Brunetti, G., et al. 2005, A\&A, 440, 867

Giovannini, G., \& Feretti, L. 2000, New Astron., 5, 335

Giovannini, G., Feretti, L., Govoni, F., Murgia, M., \& Pizzo, R. 2006, Astron. Nachr., 327, 563

Girardi, M., Escalera, E., Fadda, D., et al. 1997, ApJ, 482, 41

Girardi, M., \& Mezzetti, M. 2001, ApJ, 548, 79

Govoni, F., Feretti, L., Giovannini, G., et al. 2001, A\&A, 376, 803

Govoni, F., Markevitch, M., Vikhlinin, A., et al. 2004, ApJ, 605, 695

Gutierrez, K., \& Krawczynski, H. 2005, ApJ, 619, 161

Henry, J. P., Finoguenov, A., \& Briel, U. G. 2004, ApJ, 615, 181

Jeltema, T. E., Hallman, E. J., Burns, J. O., \& Motl, P. M. 2008, ApJ, 681, 167

Johnston-Hollitt, M., Sato, M., Gill, J. A., Fleenor, M. C., \& Brick, A.-M. 2008, MNRAS, 390, 289

Johnstone, R. M., Allen, S. W., Fabian, A. C., \& Sanders, J. S. 2002, MNRAS, 336, 299

Kassim, N. E., Clarke, T. E., Enßlin, T. A., Cohen, A. S., \& Neumann, D. M. 2001, ApJ, 559, 785

Kempner, J. C., \& David, L. P. 2004, ApJ, 607, 220

Kempner, J. C., Sarazin, C. L., \& Ricker, P. M. 2002, ApJ, 579, 236

Kempner, J. C., Sarazin, C. L., \& Markevitch, M. 2003, ApJ, 593, 291

Krivonos, R. A., Vikhlinin, A. A., Markevitch, M. L., \& Pavlinsky, M. N. 2003, Astron. Lett., 29, 425

Leborgne, J.-F., Mathez, G., Mellier, Y., et al. 1991, A\&AS, 88, 133

Leccardi, A., \& Molendi, S. 2008a, A\&A, 487, 461

Leccardi, A., \& Molendi, S. 2008b, A\&A, 486, 359

Łokas, E. L., Prada, F., Wojtak, R., Moles, M., \& Gottlöber, S. 2006, MNRAS, 366, L26

Malumuth, E. M., Kriss, G. A., Dixon, W. V. D., Ferguson, H. C., \& Ritchie, C. 1992, AJ, 104, 495

Maughan, B. J., Jones, C., Forman, W., \& Van Speybroeck, L. 2008, ApJS, 17, 117

Maurogordato, S., Cappi, A., Ferrari, C., et al. 2008, A\&A, 481, 593

Mazzotta, P., Kaastra, J. S., Paerels, F. B., et al. 2002, ApJ, 567, L37

Mazzotta, P., Edge, A. C., \& Markevitch, M. 2003, ApJ, 596, 190

McCarthy, I. G., Balogh, M. L., Babul, A., Poole, G. B., \& Horner, D. J. 2004, ApJ, 613, 811
Mercurio, A. 2004, Ph.D. Thesis, AA(Università di Trieste INAF-OAC, Napoli) Mohr, J. J., Geller, M. J., Fabricant, D. G., et al. 1996, ApJ, 470, 724

Morris, R. G., \& Fabian, A. C. 2005, MNRAS, 358, 585

Murgia, M., Govoni, F., Feretti, L., et al. 2004, A\&A, 424, 429

Oegerle, W. R., \& Hill, J. M. 2001, AJ, 122, 2858

Oegerle, W. R., Hill, J. M., \& Fitchett, M. J. 1995, AJ, 110, 32

O'Hara, T. B., Mohr, J. J., \& Guerrero, M. A. 2004, ApJ, 604, 604

O’Hara, T. B., Mohr, J. J., Bialek, J. J., \& Evrard, A. E. 2006, ApJ, 639, 64

Peres, C. B., Fabian, A. C., Edge, A. C., et al. 1998, MNRAS, 298, 416

Pizzo, R. F., de Bruyn, A. G., Feretti, L., \& Govoni, F. 2008, A\&A, 481, L91

Ponman, T. J., Sanderson, A. J. R., \& Finoguenov, A. 2003, MNRAS, 343, 331

Poole, G. B., Fardal, M. A., Babul, A., et al. 2006, MNRAS, 373, 881

Poole, G. B., Babul, A., McCarthy, I. G., Sanderson, A. J. R., \& Fardal, M. A. 2008, MNRAS, 391, 1163

Pratt, G. W., Arnaud, M., \& Pointecouteau, E. 2006, A\&A, 446, 429

Pratt, G. W., Böhringer, H., Croston, J. H., et al. 2007, A\&A, 461, 71

Reiprich, T. H., Sarazin, C. L., Kempner, J. C., \& Tittley, E. 2004, ApJ, 608, 179

Ricker, P. M., \& Sarazin, C. L. 2001, ApJ, 561, 621

Roettiger, K., Stone, J. M., \& Mushotzky, R. F. 1998, ApJ, 493, 62

Rossetti, M. 2006, Ph.D. Thesis available at http://sito

Rossetti, M., \& Molendi, S. 2010, A\&A, 510, A83

Rossetti, M., Ghizzardi, S., Molendi, S., \& Finoguenov, A. 2007, A\&A, 463, 839

Rowley, D. R., Thomas, P. A., \& Kay, S. T. 2004, MNRAS, 352, 508

Sakelliou, I., \& Ponman, T. J. 2004, MNRAS, 351, 1439

Sakelliou, I., \& Ponman, T. J. 2006, MNRAS, 367, 1409

Sanderson, A. J. R., Ponman, T. J., \& O'Sullivan, E. 2006, MNRAS, 372, 1496

Sanderson, A. J. R., Edge, A. C., \& Smith, G. P. 2009, MNRAS, 398, 1698

Sarazin, C. L. 1988, X-ray emission from clusters of galaxies, Cambridge Astrophysics Series (Cambridge: Cambridge University Press)

Sarazin, C. 2006, in Chandra Proposal, 2233

Sivanandam, S., Zabludoff, A. I., Zaritsky, D., Gonzalez, A. H., \& Kelson, D. D. 2009, ApJ, 691, 1787

Slee, O. B., \& Roy, A. L. 1998, MNRAS, 297, L86

Slee, O. B., Roy, A. L., Murgia, M., Andernach, H., \& Ehle, M. 2001, AJ, 122 , 1172

Smith, G. P., Kneib, J.-P., Smail, I., et al. 2005, MNRAS, 359, 417

Snowden, S. L., Mushotzky, R. F., Kuntz, K. D., \& Davis, D. S. 2008, A\&A, 478,615

Sun, M., Murray, S. S., Markevitch, M., \& Vikhlinin, A. 2002, ApJ, 565, 867

Sun, M., Jones, C., Forman, W., Vikhlinin, A., Donahue, M., \& Voit, M. 2007, ApJ, 657, 197

Takizawa, M., Sarazin, C. L., Blanton, E. L., \& Taylor, G. B. 2003, ApJ, 595, 142

Tustin, A. W., Geller, M. J., Kenyon, S. J., \& Diaferio, A. 2001, AJ, 122, 1289

Venturi, T., Bardelli, S., Zagaria, M., Prandoni, I., \& Morganti, R. 2002, A\&A, 385,39

Venturi, T., Giacintucci, S., Brunetti, G., et al. 2007, A\&A, 463, 937

Venturi, T., Giacintucci, S., Dallacasa, D., et al. 2008, A\&A, 484, 327

Vikhlinin, A., Markevitch, M., \& Murray, S. S. 2001, ApJ, 551, 160

Vikhlinin, A., Markevitch, M., Murray, S. S., et al. 2005, ApJ, 628, 655

Vikhlinin, A., Burenin, R., Forman, W. R., et al. 2007, in Heating versus Cooling in Galaxies and Clusters of Galaxies, ed. H. Böhringer, G. W. Pratt, A. Finoguenov, \& P. Schuecker, 48

Voit, G. M. 2005, Adv. Space Res., 36, 701

Wise, M. W., McNamara, B. R., \& Murray, S. S. 2004, ApJ, 601, 184

Zwicky, F., Herzog, E., \& Wild, P. 1968, Catalogue of galaxies and of clusters of galaxies, ed. F. Zwicky, E. Herzog, \& P. Wild 